\title{
Optical and infrared properties of tetramethyltetraselenafulvalene [(TMTSF)2X] and tetramethyltetrathiafulvalene [(TMTTF)2X] compounds
}

\author{
Jacobsen, Claus Schelde; Tanner, D. B.; Bechgaard, K.
}

Published in:

Physical Review B

Link to article, DOI:

10.1103/PhysRevB.28.7019

Publication date:

1983

Document Version

Publisher's PDF, also known as Version of record

Link back to DTU Orbit

Citation (APA):

Jacobsen, C. S., Tanner, D. B., \& Bechgaard, K. (1983). Optical and infrared properties of

tetramethyltetraselenafulvalene [(TMTSF)2X] and tetramethyltetrathiafulvalene [(TMTTF)2X] compounds.

Physical Review B, 28(12), 7019- 7032. https://doi.org/10.1103/PhysRevB.28.7019

\section{General rights}

Copyright and moral rights for the publications made accessible in the public portal are retained by the authors and/or other copyright owners and it is a condition of accessing publications that users recognise and abide by the legal requirements associated with these rights.

- Users may download and print one copy of any publication from the public portal for the purpose of private study or research.

- You may not further distribute the material or use it for any profit-making activity or commercial gain

- You may freely distribute the URL identifying the publication in the public portal 


\title{
Optical and infrared properties of tetramethyltetraselenafulvalene [(TMTSF) ${ }_{2} X$ ] and tetramethyltetrathiafulvalene $\left[(\mathrm{TMTTF})_{2} \mathrm{X}\right]$ compounds
}

\author{
C. S. Jacobsen \\ Physics Laboratory 3, Technical University of Denmark, DK-2800 Lyngby, Denmark \\ D. B. Tanner \\ Physics Department, University of Florida, Gainesville, Florida 32611 \\ K. Bechgaard \\ H. C. Orsted Institute, DK-2100 Copenhagen, Denmark
}

(Received 5 July 1983)

\begin{abstract}
The electronic structure of the organic conductors bis-tetramethyltetraselenafulvalene- $X$ $\left[(\mathrm{TMTSF})_{2} X\right]$ and bis-tetramethyltetrathiafulvalene- $X\left[(\mathrm{TMTTF})_{2} X\right]$ has been investigated by means of polarized optical and infrared reflectance measurements. Analysis of plasma edges in reflectance is used to extract information on transfer integrals. Measurements of infrared reflectance provide information on the energy of charge-transfer processes and on electron-molecular vibration coupling. Far-infrared measurements allow comparison with low-frequency transport properties, and give clues to the transport mechanisms. The main results may be summarized as follows: The (TMTSF) ${ }_{2} \mathrm{X}$ class of materials has chain-axis transfer integrals of order $0.25 \mathrm{eV}$ at $300 \mathrm{~K}$ and 0.28 $\mathrm{eV}$ at $30 \mathrm{~K}$. The $b$-axis transfer integral is found to vary from 18 to $24 \mathrm{meV}$ for different $X$. The $(\text { TMTTF })_{2} X$ class has a chain-axis transfer integral of the order $0.18-0.20 \mathrm{eV}$. No $b$-axis plasma edge is observable. The infrared conductivity spectra of the materials consist of a broad electronic band with superimposed vibrational fine structure. The band is centered at $300 \mathrm{~cm}^{-1}$ in the best (TMTSF) ${ }_{2} X$ conductors and at $2200 \mathrm{~cm}^{-1}$ in $(\mathrm{TMTTF})_{2} \mathrm{PF}_{6}$, an organic conductor of moderate conductivity. The electron-molecular vibration coupling constants for TMTSF and TMTTF appear to be qualitatively similar to those of TTF (tetrathiafulvalene). A new feature is the observation of considerable coupling to modes involving methyl groups, suggesting that a sizable charge density is located near these groups. The electronic band in (TMTSF) ${ }_{2} \mathbf{P F}_{6}$ sharpens at low temperature, and a pseudogap at $180 \mathrm{~cm}^{-1}$ is formed at temperatures above the metal-insulator transition. This behavior is discussed in terms of a possible spin-density-wave contribution to the conductivity. The spin-density-wave amplitude is estimated to be $0.02 \mu_{B}$.
\end{abstract}

\section{INTRODUCTION}

Organic conductors based on the donor molecules TMTSF (tetramethyltetraselenafulvalene) and TMTTF (tetramethyltetrathiafulvalene) combined in a 2:1 ratio with various monovalent, inorganic ions (such as $\mathrm{PF}_{6}{ }^{-}$, $\mathrm{AsF}_{6}^{-}, \mathrm{ClO}_{4}^{-}, \mathrm{ReO}_{4}^{-}, \mathrm{Br}^{-}$, etc.) are of great current interest. ${ }^{1,2}$ The TMTSF group comprises mostly good organic metals, typically with conductivities $\sigma(300 \mathrm{~K})=600$ $\Omega^{-1} \mathrm{~cm}^{-1}$, and $\sigma(T) \sim T^{-2}$ (Ref. 3). Observed ground states include superconductivity ${ }^{4,5}\left(T_{c} \cong 1 \mathrm{~K}\right.$, ambient or small hydrostatic pressure), antiferromagnetism, ${ }^{6-8}$ and nonmagnetic insulators. ${ }^{9}$ The TMTTF group contains generally organic metals in the intermediate class $\left[\sigma(300 \mathrm{~K})=1-100 \Omega^{-1} \mathrm{~cm}^{-1}\right]$ with broad conductivity maxima and semiconducting behavior at low temperatures. ${ }^{10}$ The application of high pressure induces metallic behavior $^{11}$ and makes the materials look more like the selenium analogs, although superconductivity has not been safely identified. In both classes it is found that counterions with lower than octahedral symmetry often perturb the electronic properties through order-disorder transfor- mations, which change the crystal symmetry. ${ }^{12}$

Several features are identical for all materials in the two groups. Most important are the crystal structure and the band filling. The triclinic unit cell ${ }^{13,14}$ contains one anion and two donor molecules with a slipped overlap, qualitatively like that of the TTF stack in TTF-TCNQ (tetrathiafulvalene-tetracyanoquinodimethane). ${ }^{15}$ However, in contrast to TTF-TCNQ, the stacking axis $(a)$ is almost normal to the molecular plane, resulting in a zigzag type of stack. Neighbor stacks interact through short intermolecular separations forming sheets of molecular stacks (along the $b$ axis) alternating with sheets of anions (along the $c$ axis). Thus a highly anisotropic band structure results.

The basic one-electron, one-dimensional band structure is sketched in Fig. 1. The conduction band is a half-filled hole band. The small gap at the zone edge follows from a small dimerization associated with the zigzag structure. The size of this gap (as estimated from intermolecular distances) is normally assumed to vary strongly from material to material and may have implications for superconductivity, etc. ${ }^{16}$ 


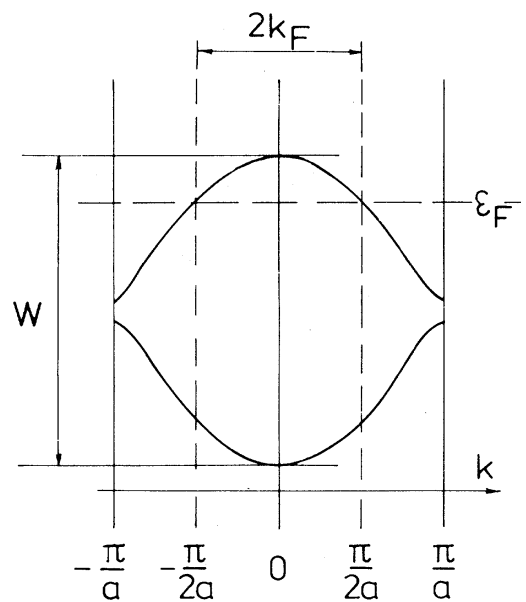

FIG. 1. One-electron, one-dimensional band structure of (TMTSF) $)_{2} X$ and (TMTTF $)_{2} X$ in the stacking direction. Note that the lattice constant $a$ comprises two organic molecules.

Attractive features which make physical interpretation simpler include the presence of one kind of stack only, the virtual absence of mixing between excitations in the molecular plane and excitations in the stacking direction (except for the anomalous vibrational features discussed below), and a charge density given by stoichiometry.

The purpose of the present paper is to describe experiments on optical and infrared properties, which we have made over the last few years. We shall discuss the implications of these studies for band structure, electronmolecular-vibration (emv) coupling, phase transitions, and conductivity mechanism. Preliminary results have previously been reported in Refs. 17 and 18. Related to our work has been the studies by Kikuchi et al. ${ }^{19}$ on the overall optical properties of (TMTSF) ${ }_{2} \mathrm{ClO}_{4}$, the work on vibrational assignments and emv coupling by Bozio and co-workers, ${ }^{20,21}$ and far-infrared investigations on $(\mathrm{TMTSF})_{2} \mathrm{ClO}_{4}$ by $\mathrm{Ng}$ et al. ${ }^{22,23}$ and Challener et al. ${ }^{24}$ Helberg ${ }^{25}$ has conducted studies concerning the orientation of the principal axes in the visible range.

The plan of the paper is the following. First, we discuss experimental procedures. Next, we address the electronic structure of these materials for $a, b$, and $c$ polarizations. We then describe in some detail the signatures of emv coupling and the distribution of oscillator strength in the chain-axis direction. For the single case [(TMTSF) $\left.{ }_{2} \mathbf{P F}_{6}\right]$ where far-infrared spectra have been obtained, we give a detailed analysis of the data and discuss our results in relation to the de transport properties.

\section{EXPERIMENTAL}

Single crystals of the materials were prepared electrochemically as described elsewhere. ${ }^{3}$ Microanalysis showed 2:1 stoichiometry within experimental limits (0.5\%). All samples were elongated in the $a$-axis direction and had most often reasonable quality (001) faces. Measurements have been done for the electric field along the stacking $a$ axis direction, and along the $b^{\prime}$ axis, which designates the direction in the $a-b$ plane, which is perpendicular to the $a$ axis. A few samples have been investigated in unidentified faces in directions approximately perpendicular to $a$ and $b^{\prime}$.

In a triclinic crystal nothing can be said a priori about the optical axes. However, the observed conductivity anisotropies ${ }^{3}$ suggest that $a$ and $b^{\prime}$ are principal axes at low frequencies, where charge-transfer excitations dominate, i.e., in the infrared range. Helberg ${ }^{25}$ has found that $a$ is a principal axis through the visible as well, although the other axes rotate in this range. Thus the measured $b^{\prime}$-axis spectra are not representative of a principal axis in the entire frequency range.

Owing to the opaque nature of the crystals, all measurements were done as reflectance. As reflectance standard we used evaporated gold or aluminum giving an accuracy of order $1 \%$ on the absolute value.

Various instruments were employed. The far infrared $\left(10-700 \mathrm{~cm}^{-1}\right)$ was covered with a Michelson interferometer, combined with a wire grid polarizer and a cooled bolometer detector. Two Perkin-Elmer grating monochromators (PE-16U and PE-98) provided radiation in the $(350-20000)-\mathrm{cm}^{-1}$ range. Some measurements in the $(5000-30000)-\mathrm{cm}^{-1}$ range were done with a fused quartz prism monochromator (PE-98). A Perkin-Elmer polarizer (gold grid on $\mathrm{AgBr}$ ) was used from 350-5000 $\mathrm{cm}^{-1}$, while Glan-Thompson prisms and plastic (dichroic) polarizers were used at higher frequencies. A variety of detectors has been employed including thermocouples, $\mathrm{PbS}$ cells, Golay cells, and photomultipliers. As radiation sources, a mercury arc, a Globar, a tungsten filament lamp, and a xenon high-pressure arc have been used for the far infrared, intermediate infrared, near infrared, and visible to near ultraviolet, respectively.

Most measurements were done on single crystals, a few $\mathrm{mm}$ long and up to $0.5 \mathrm{~mm}$ wide. However, for farinfrared and in some cases intermediate-infrared measurements, mosaics of optically aligned crystals were constructed to ensure an acceptable $S / N$ ratio. After the primary reflectance measurements these mosaics were covered with gold and the measurements repeated. In this way the effective filling factor of the mosaic can be reliably estimated, and simultaneously undesired diffraction or interference effects of the mosaic are revealed. Such effects were only observed in the very far infrared, for $\vec{E} \| b^{\prime}$, where the small crystal dimension is comparable to the wavelength of the radiation. In the data presentation, it has been assumed that the gold-covered mosaic provides a usable reference spectrum.

Low-temperature spectra were obtained by thermally anchoring the sample to a controlled cold finger. Radiation shields with suitable holes were used. However, in all cases a considerable amount of $300-\mathrm{K}$ radiation was absorbed in the sample. To estimate the actual sample temperature, a TTF-TCNQ crystal was placed in the sample position, and the well-known resistivity versus temperature of this material ${ }^{26}$ was used as a calibration. The reflectance and absorbance properties of TTF-TCNQ (Ref. 27) are quite comparable to those of the (TMTSF) ${ }_{2} \mathrm{X}$ materials in the range of $300-\mathrm{K}$ radiation. It has been found that with the mounting technique employed sample temperatures cannot be lowered below about $25 \mathrm{~K}$. 


\section{ELECTRONIC STRUCTURE OF THESE COMPOUNDS}

In Fig. 2 is presented typical near-infrared-visiblereflectance spectra of (TMTSF) ${ }_{2} \mathrm{AsF}_{6}$ and (TMTTF) ${ }_{2} \mathrm{PF}_{6}$ taken at $300 \mathrm{~K}$ for polarizations along $a$ and $b^{\prime}$. The broad features are identical to those found in many other organic conductors, as, for example, TTF-TCNQ (Ref. 28): The stacking direction $(a)$ displays a sharp drop in the near infrared with a minimum at 8000 and $7600 \mathrm{~cm}^{-1}$ for the two materials, respectively. The position agrees with earlier studies on (TMTSF) ${ }_{2} \mathrm{PF}_{6},{ }^{17}$ (TMTSF) ${ }_{2} \mathrm{ClO}_{4},{ }^{19}$ and on polycrystalline samples of the (TMTTF $)_{2} X$ salts. $^{10}$ This reflectance edge is associated with the plasma behavior of the conduction electrons. From the minimum and up through the visible no transitions can be identified. In contrast, TTF-TCNQ displays a number of bands for polarization along the chain axis. ${ }^{28}$

\section{A. Molecular excitations in the transverse direction}

For $\overrightarrow{\mathrm{E}}|| b^{\prime}$ the reflectance is low and rather dispersionless throughout the range: Here the materials are highly anisotropic. In the visible at least two absorption bands can be seen. In (TMTTF) ${ }_{2} \mathrm{PF}_{6}$ there is a band at 17000 $\mathrm{cm}^{-1}$ and a shoulder at $22000 \mathrm{~cm}^{-1}$. The bands in (TMTSF) ${ }_{2} X$ can be seen more clearly in Fig. 3(a), where two polarizations perpendicular to the chain axis in (TMTSF) ${ }_{2} \mathrm{SbF}_{6}$ are shown, and in Fig. 3(b) where the temperature dependence for (TMTSF) ${ }_{2} \mathrm{AsF}_{6}$ along $b^{\prime}$ is presented. There are two distinct absorption bands at approximately 15000 and $18000 \mathrm{~cm}^{-1}$ and a rise towards another band above $25000 \mathrm{~cm}^{-1}$. There may be a weak band near $10000 \mathrm{~cm}^{-1}$. Similar bands were found by $\mathrm{Ki}$ kuchi et al. ${ }^{19}$ in (TMTSF) ${ }_{2} \mathrm{ClO}_{4}\left(b^{\prime}\right)$ and must therefore be characteristic for the TMTSF stacks.

An interesting feature is that the $18000-\mathrm{cm}^{-1}$ band is strongest in the $b^{\prime}$ polarization, while the $15000-\mathrm{cm}^{-1}$ band is strongest in the other polarization. The long axis of the TMTSF molecule is about $65^{\circ}$ off the $b^{\prime}$ axis. ${ }^{13}$ Thus it appears that the $15000-\mathrm{cm}^{-1}$ transition is polar-

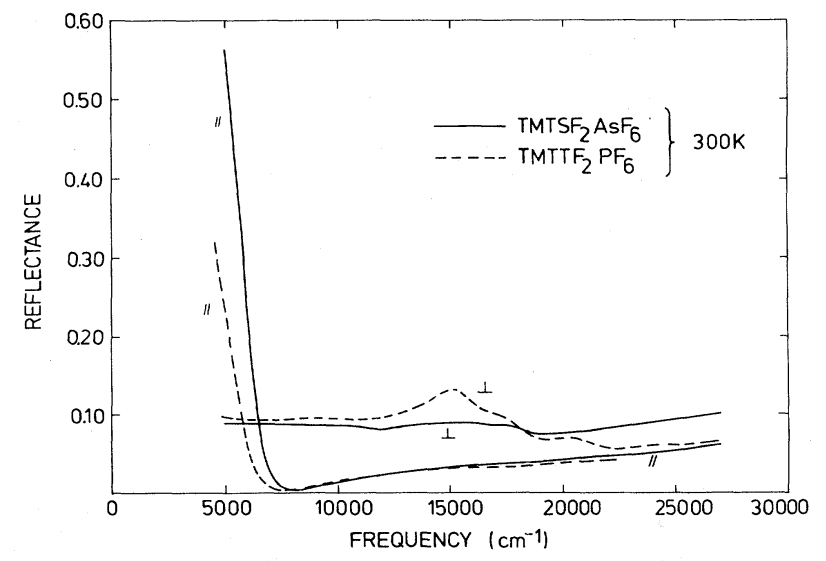

FIG. 2. Polarized room-temperature reflectance of (TMTSF) $)_{2} \mathrm{AsF}_{6}$ and (TMTTF) ${ }_{2} \mathrm{PF}_{6}$ from 5000 to $27000 \mathrm{~cm}^{-1}$. The stacking axis $a$ corresponds to $\|$, while $\perp$ is the $b^{\prime}$ direction.
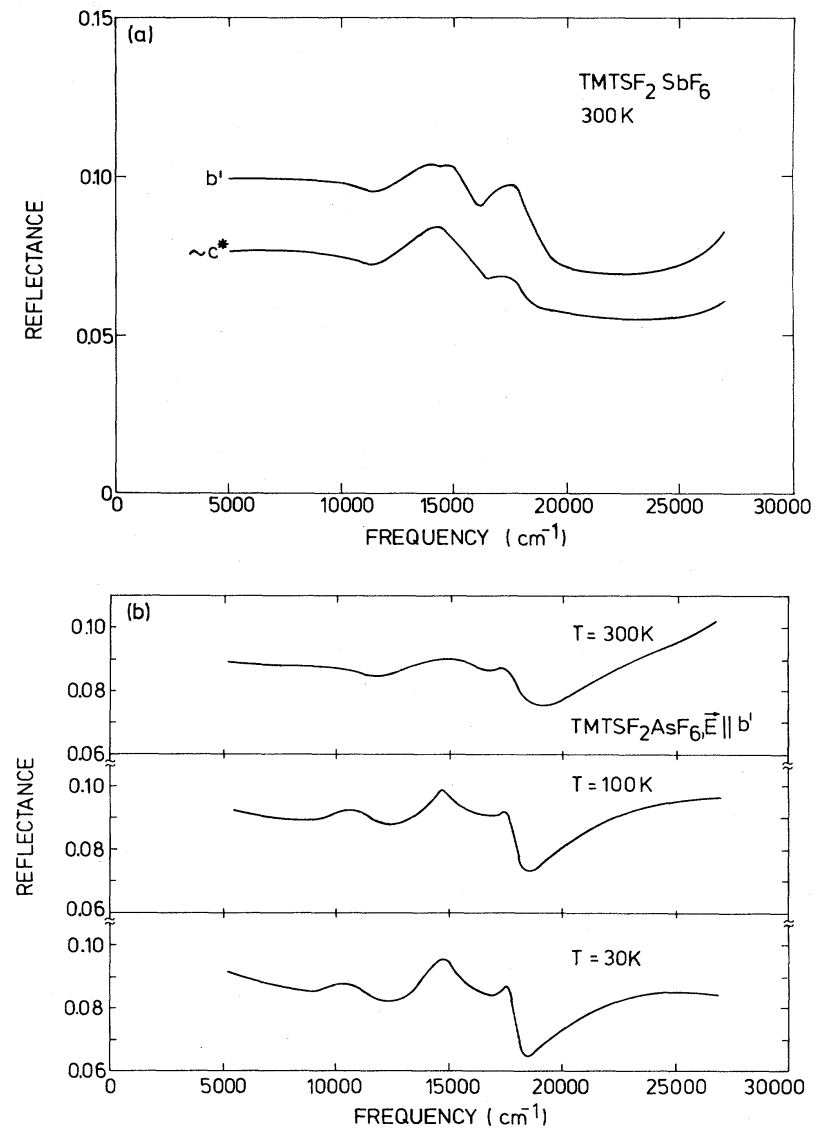

FIG. 3. Polarized reflectance of (TMTSF) ${ }_{2} X$ perpendicular to chain axis. (a) (TMTSF) ${ }_{2} \mathrm{SbF}_{6}$ for two different polarizations at $T=300 \mathrm{~K}$, and (b) (TMTSF) ${ }_{2} \mathrm{AsF}_{6}$ for $\overrightarrow{\mathrm{E}}|| b^{\prime}$ at $T=300,100$, and $30 \mathrm{~K}$. The frequency range is $5000-27000 \mathrm{~cm}^{-1}$.

ized along the long axis of the TMTSF molecule, while the $18000-\mathrm{cm}^{-1}$ band is polarized along the short axis. The main effect of lowering the temperature is a sharpening of the bands.

According to the work by Zahradnik et al. ${ }^{29}$ on $\mathrm{TTF}^{+}$ and by Gleiter et $a l .{ }^{30}$ on the neutral molecules, the conduction electron resides in a $b_{1 u}$ orbital with lowest allowed transitions to and from $b_{2 g}$ and $b_{3 g}$ orbitals. Solution spectra indicate the presence of a weak absorption band at lower energy than the others, tentatively identified as a forbidden $b_{1 u} \rightarrow a_{u}$ transition allowed by vibronic coupling. If the $10000-\mathrm{cm}^{-1}$ band is real, it may be due to the latter transition, and thus opens the possibility of an excited level about $1.2 \mathrm{eV}$ from the bottom of the conduction band. This is comparable to the one-electron bandwidth estimated below. The $15000-\mathrm{cm}^{-1}$ excitation should then be to or from a $b_{3 g}$ level, while the 18000 $\mathrm{cm}^{-1}$ band corresponds to a transition to or from a $b_{2 g}$ level. These assignments are based on the observed polarizations. $^{30}$

We note that the spectrum is qualitatively different from those of (TMTTF) ${ }_{2} \mathrm{PF}_{6}$ (presented here), (TMTTF) Br $r_{x},{ }^{31}$ and (TSF) Br $r_{x},{ }^{31}$ which all show a strong 
band in (15000-18000)- $\mathrm{cm}^{-1}$ range and a weak shoulder at $22000 \mathrm{~cm}^{-1}$. Thus it is conceivable that the electronic structure of TMTSF may deviate in a qualitative way from other tetrachalcogenafulvalenes. This point deserves further clarification.

\section{B. Chain-axis transfer integral}

Returning now to the chain-axis polarization, the absence of bands in the visible is a clear consequence of the orientation of the molecules. The transition dipoles of low-lying intramolecular transitions are close to perpendicular to the stacking axis. However, any charge-transfer excitation along the stack would be observed. It seems that there is only one such charge-transfer band, i.e., that associated with the plasma edge. There is no sign of localized excitations corresponding to double occupancy on TMTSF molecules (the so-called $U$ band). Such excitations are seen in several TTF halides, ${ }^{31}$ but may be expected to be very weak when the carrier density is low. ${ }^{32}$

In Fig. 4 we show the temperature dependence of the $\overrightarrow{\mathbf{E}} \| a$ spectrum of (TMTSF) ${ }_{2} \mathrm{AsF}_{6}$. The only significant change is a small blue shift $\left(\cong 500 \mathrm{~cm}^{-1}\right)$ of the plasma edge on cooling. A similar shift has been observed in (TMTSF) ${ }_{2} \mathrm{ClO}_{4}$ by Kikuchi et al., ${ }^{19}$ and is quite similar to the shift of the edge in TTF-TCNQ. ${ }^{33}$ The shift may be associated with thermal contraction, which results in an increase in bandwidth.

The near-infrared chain-axis reflectance of organic conductors is customarily analyzed in terms of the Drude model for the dielectric function:

$$
\widetilde{\epsilon}(\omega)=\epsilon_{\infty}-\frac{\omega_{p}^{2}}{\omega(\omega+i \Gamma)} .
$$

$\omega_{p}$ is the bare plasma frequency, $\epsilon_{\infty}$ is the background dielectric constant, and $\Gamma$ is a relaxation rate. This model is indeed found to represent the TMTTF and TMTSF materials very well in the vicinity of the plasma edge, in part because unlike the TCNQ salts, there are no absorption bands near the edge to take into account.

Assuming a one-electron band, $\omega_{p}$ is given by ${ }^{34}$

$$
\omega_{p}^{2}=\left(e^{2} / \hbar^{2} \epsilon_{0}\right) \sum_{\mathrm{BZ}}\left[\frac{\partial^{2} \epsilon_{k}}{\partial k_{\mu}^{2}}\right] f\left(\epsilon_{k}\right),
$$

where BZ represents the Brillouin zone, $f\left(\epsilon_{k}\right)$ is the Fermi-Dirac occupation number, and $\partial^{2} \epsilon_{k} / \partial k_{\mu}^{2}$ indicates a derivative along the polarization of the electric field. For a one-dimensional tight-binding band with transfer integral $t$, intermolecular spacing $d$, volume per molecule

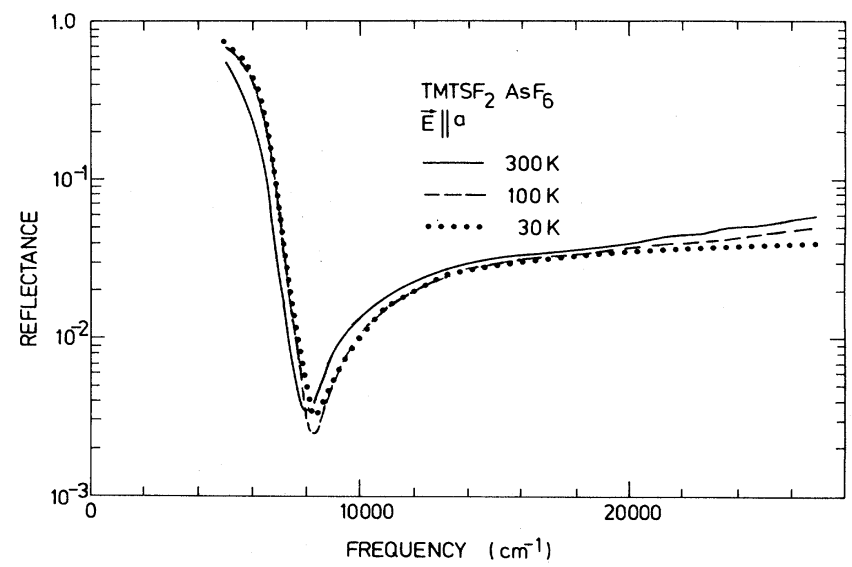

FIG. 4. Chain-axis reflectance of (TMTSF) ${ }_{2} \mathrm{AsF}_{6}$ at $T=300$, 100 , and $30 \mathrm{~K}$. The frequency range is $5000-27000 \mathrm{~cm}^{-1}$. Notice the logarithmic reflectance scale.

$V_{m}$, and number of carriers per molecule $\rho$, Eq. (2) yields (at $T=0$ )

$$
\omega_{p}^{2}=\frac{4 t d^{2} e^{2}}{\pi \epsilon_{0} \hbar^{2} V_{m}} \sin \left(\frac{\pi \rho}{2}\right) .
$$

Here $d=a / 2$ and $\rho=0.5$. Thus the dimerization associated with the zigzag structure is neglected, making the resulting transfer integral an average value.

Table I gives Drude parameters and transfer integrals for $(\mathrm{TMTSF})_{2} \mathrm{AsF}_{6}$ at three temperatures, and for (TMTSF) ${ }_{2} \mathrm{PF}_{6}$ and $(\mathrm{TMTTF})_{2} \mathrm{PF}_{6}$ at room temperature. The Drude parameters have been obtained by fitting Eq. (1) to the logarithm of reflectance. In this way the plasma minimum and the low values of reflectance above the edge are well reproduced, ensuring a precise determination of $\omega_{p}$ and $\epsilon_{\infty}$. The results for $(\mathrm{TMTSF})_{2} \mathrm{AsF}_{6}$ and (TMTSF) ${ }_{2} \mathrm{PF}_{6}$ compares very well with those reported by Kikuchi et al. ${ }^{19}$ on (TMTSF) ${ }_{2} \mathrm{ClO}_{4}$. Other materials like (TMTSF) ${ }_{2} \mathrm{SbF}_{6}$ and (TMTSF) ${ }_{2} \mathrm{ReO}_{4}$ are found to have very similar edge positions. Thus it appears that the whole (TMTSF) ${ }_{2} X$ group has nearly the same $\omega_{p}=9900$ $\mathrm{cm}^{-1} \pm 2 \%$ at room temperature, with a $5 \%$ increase on cooling. In TTF-TCNQ $\omega_{p}$ increases about $10 \%$ on cooling, ${ }^{33}$ but this is partly due to a change in carrier density. Eliminating this carrier density change the shifts are almost identical.

Estimates of transfer integrals based on Eq. (3) are generally found to be reliable for highly conducting organic salts in the sense that they vary consistently with expecta-

TABLE I. Drude parameters and transfer integrals for the chain axis.

\begin{tabular}{lccccc}
\hline \hline Material & $T(\mathrm{~K})$ & $\epsilon_{\infty}$ & $\omega_{p}\left(\mathrm{~cm}^{-1}\right)$ & $\Gamma\left(\mathrm{cm}^{-1}\right)$ & $t_{a}(\mathrm{eV})$ \\
\hline$(\mathrm{TMTSF})_{2} \mathrm{AsF}_{6}$ & 300 & 2.56 & 9940 & 1230 & 0.25 \\
$(\mathrm{TMTSF})_{2} \mathrm{AsF}_{6}$ & 100 & 2.52 & 10270 & 1030 & 0.27 \\
$(\mathrm{TMTSF})_{2} \mathrm{AsF}_{6}$ & 30 & 2.55 & 10470 & 1160 & 0.28 \\
$(\mathrm{TMTSF})_{2} \mathrm{PF}_{6}$ & 300 & 2.42 & 9700 & 1520 & 0.25 \\
$(\mathrm{TMTTF})_{2} \mathrm{PF}_{6}$ & 300 & 2.50 & 8860 & 1380 & 0.20 \\
\hline \hline
\end{tabular}


tions from material to material, ${ }^{31}$ with varying temperature, ${ }^{33}$ and so forth. The value found for (TMTSF) ${ }_{2} X$, $t_{a} \cong 0.25 \mathrm{eV}$ at room temperature, is surprisingly large. However, estimates of this quantity based on thermoelectric power, which depends on both the band structure and the details in the scattering mechanism, give $t_{a} \cong 0.35 \mathrm{eV}$, assuming acoustic one-phonon scattering. ${ }^{35}$ Also a recent reanalysis of TMTSF-DMTCNQ (dimethyltetracyanoquinodimethane), ${ }^{36}$ yields $t \cong 0.25 \mathrm{eV}$ for the TMTSF stack. This stack is regular, with a slipped overlap almost identical to that in (TMTSF $)_{2} X$.

A relevant material for comparison is TSF-TCNQ. TSF (tetraselenafulvalene) is the parent molecule of TMTSF (i.e., with no methyl groups). The overlap pattern of TSF molecules in TSF-TCNQ (Ref. 37) is similar to that of TMTSF in (TMTSF) ${ }_{2} X$. The interplanar distances are 3.52 and $3.65 \AA$, respectively. Thus if the methyl groups had no drastic effects on the orbital, the TSF stack would be expected to have a slightly larger $t$ than a TMTSF stack. In contrast, Welber et al. ${ }^{38}$ find $t=0.15 \mathrm{eV}$ for the TSF stack. Although this value depends on an estimate for the TCNQ stack in this material, to make it much larger would be inconsistent with the data. The main conclusion of this discussion is that the attached methyl groups do indeed significantly perturb the highest occupied molecular orbital in TMTSF in a way to enhance the stacking overlap. Molecular vibration studies (see below) indicate that charge may be displaced towards the methyl groups.

The results for (TMTTF) ${ }_{2} \mathrm{PF}_{6}$ given in Table I should be regarded with reservation. The material is not highly conducting $\left[\sigma(300 \mathrm{~K}) \simeq 20 \Omega^{-1} \mathrm{~cm}^{-1}\right] .{ }^{10}$ Also infrared measurements, reported below, indicate that the chargetransfer band is centered around $2200 \mathrm{~cm}^{-1}$, which is not vanishing compared to $\omega_{p}$. Fitting to a Drude-Lorentz model

$$
\widetilde{\boldsymbol{\epsilon}}(\omega)=\epsilon_{\infty}-\frac{\omega_{p}^{2}}{\left(\omega^{2}-\omega_{\mathrm{CT}}^{2}\right)+i \omega \Gamma},
$$

where $\hbar \omega_{\mathrm{CT}}$ is the energy of the dominant charge-transfer excitation, yields $\omega_{p} \cong 8600 \mathrm{~cm}^{-1}$, which with the model of Eq. (3) gives $t \simeq 0.18 \mathrm{eV}$. However, this model is difficult to reconcile with a low dc conductivity and a chargetransfer band at such a high frequency. So a considerable uncertainity in the estimate of $t$ for (TMTTF) ${ }_{2} X$ remains.

\section{Transfer integral perpendicular to chain axis}

In this section we present and discuss data relevant to the so-called transverse plasma edge in (TMTSF) ${ }_{2} X{ }^{17,18}$ Figure 5 gives the $b^{\prime}$-axis reflectance of (TMTSF) ${ }_{2} \mathrm{PF}_{6}$ at 300 and $25 \mathrm{~K}$ from 0 to $1800 \mathrm{~cm}^{-1}$. At $300 \mathrm{~K}, R$ drops rapidly from unity and becomes rather flat above 500 $\mathrm{cm}^{-1}$. The peaks at 560 and $835 \mathrm{~cm}^{-1}$ are due to vibrational modes in the $\mathrm{PF}_{6}{ }^{-}$ion. ${ }^{39}$ At $25 \mathrm{~K}$ the overall reflectance remains rather high up to $700 \mathrm{~cm}^{-1}$, while it drops rapidly to low values on the high side of the split $\mathrm{PF}_{6}-$ bands. The rise in reflectance occurs gradually with cooling; there is no indication of a sudden transition. A minimum is located around $1200-1400 \mathrm{~cm}^{-1}$, while at higher frequencies $R$ reaches a level of about $9 \%$ with no significant structure until the visible. In addition to these overall features, there is considerable vibrational fine structure in the far infrared.

We understand this behavior as arising from metallic properties in the $b^{\prime}$ direction: At $300 \mathrm{~K}$ the plasma edge is overdamped, while at $25 \mathrm{~K}$ it is reasonably sharp, although with a lot of superimposed features from molecular vibrations. Figure 6 presents the corresponding frequency-dependent conductivity as obtained from dispersion analysis. ${ }^{34}$ As expected $\sigma_{b}(\omega)$ is rather flat at room temperature. The extrapolated dc level is about 20 $\Omega^{-1} \mathrm{~cm}^{-1}$, a bit higher than the reported ${ }^{40}$ value of 4 $\Omega^{-1} \mathrm{~cm}^{-1}$. At $25 \mathrm{~K} \sigma_{b}(\omega)$ rises below $1000 \mathrm{~cm}^{-1}$, reaching a level near dc of $140 \Omega^{-1} \mathrm{~cm}^{-1}$, again larger than the dc conductivity, ${ }^{40}$ which is $30 \Omega^{-1} \mathrm{~cm}^{-1}$. It seems conceivable that the dc conductivity is inhibited by macroscopic defects.

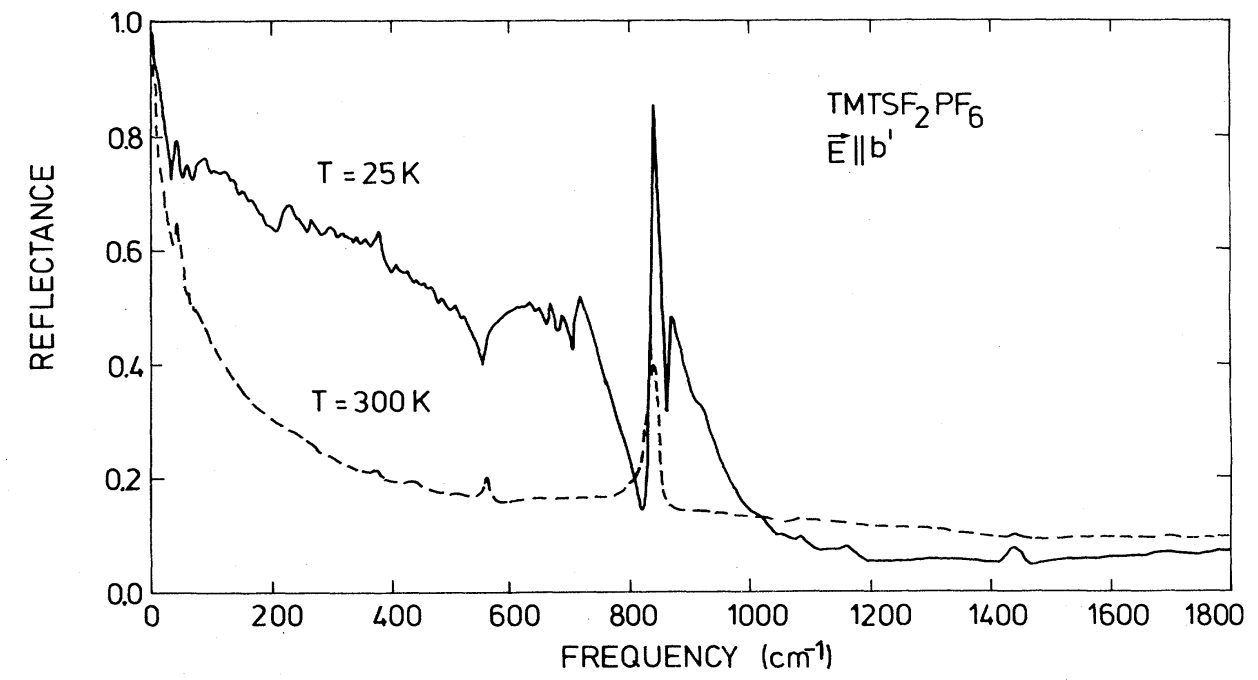

FIG. 5. Polarized reflectance for $\overrightarrow{\mathrm{E}}|| b^{\prime}$ of $(\mathrm{TMTSF})_{2} \mathrm{PF}_{6}$ at $T=25$ and $300 \mathrm{~K}$. The frequency range is $10-1800 \mathrm{~cm}^{-1}$. 


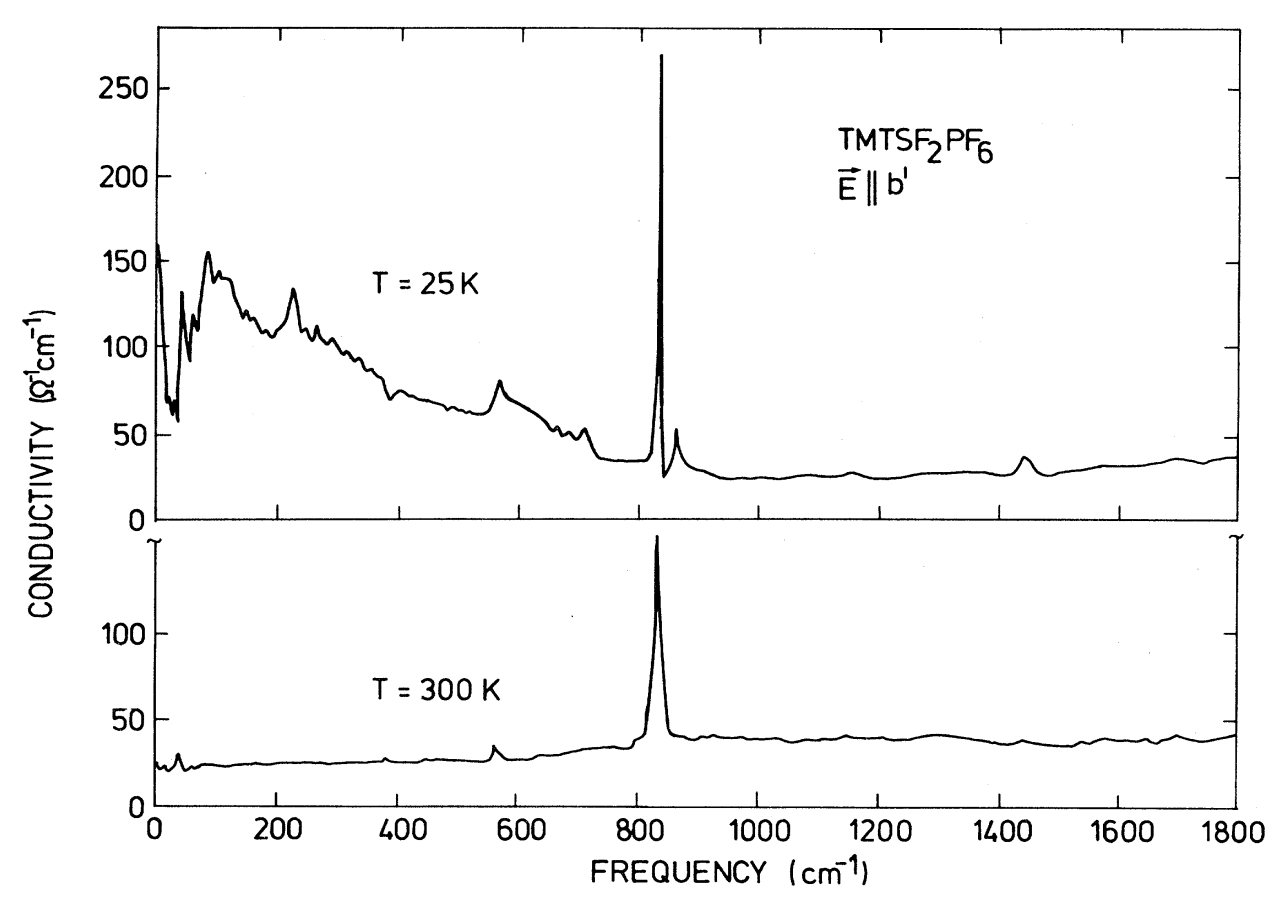

FIG. 6. Frequency-dependent conductivity of (TMTSF) $)_{2} \mathrm{PF}_{6}$ for $\overrightarrow{\mathrm{E}}|| b^{\prime}$, as obtained by dispersion analysis of reflectance data. Data are given for $T=25$ and $300 \mathrm{~K}$ in the frequency range $10-1800 \mathrm{~cm}^{-1}$.

At both 25 and $300 \mathrm{~K}$ we note that most of the vibrational lines appear as superimposed bands with no clear evidence for coupling effects. One exception may be the dip in $\sigma_{b}(\omega)$ below $100 \mathrm{~cm}^{-1}$ at $25 \mathrm{~K}$. Apart from this, there is no evidence for a gap along $b^{\prime}$. In order to verify the interpretation of the $b^{\prime}$-axis spectra, we have performed a series of measurements on other materials.

Figure 7 shows the $b^{\prime}$-axis reflectance for

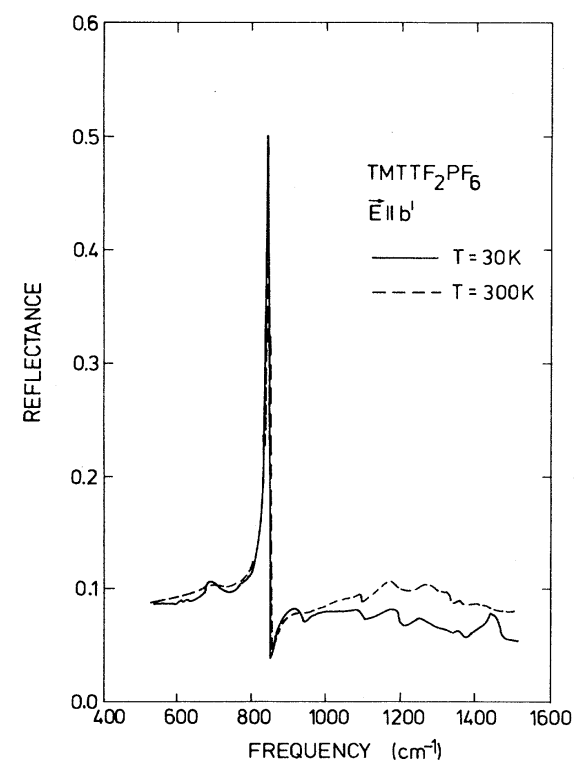

FIG. 7. Polarized reflectance for $\overrightarrow{\mathrm{E}}|| b^{\prime}$ of $(\mathrm{TMTTF})_{2} \mathrm{PF}_{6}$ at $T=30$ and $300 \mathrm{~K}$. The frequency range is $500-1500 \mathrm{~cm}^{-1}$.
(TMTTF $)_{2} \mathrm{PF}_{6}$ at 300 and $30 \mathrm{~K}$ in the range $600-1500$ $\mathrm{cm}^{-1}$. The only spectral feature is the $835-\mathrm{cm}^{-1} \mathrm{PF}_{6}-$ line; there is no rise in reflectance level as temperature is lowered. A similar check has been done on (TMTTF) ${ }_{2} \mathrm{Br}$, which is the best metal in the TMTTF group, down to 300 $\mathrm{cm}^{-1}$; again no sign of metallic behavior is seen. These data show that the high $b^{\prime}$-axis reflectance level in (TMTSF) ${ }_{2} \mathbf{P F}_{6}$ occurs only for the TMTSF chain system. Inspection of the crystal structure ${ }^{13}$ suggests that the plasma edge is associated with transfer of charge from TMTSF stack to neighbor TMTSF stack. The transfer is mediated by a rather high transfer integral. A change in the donor molecule from selenium to sulfur apparently diminishes the transfer integral considerably, so that the rise in reflectance is unobservable in the measured range.

In the third crystallographic direction the transfer integral should be negligible and no rise in $R$ is expected. Figure 8 shows the low-temperature reflectance of (TMTSF) ${ }_{2} \mathrm{AsF}_{6}$ along $b^{\prime}$ and along the direction, which is approximately perpendicular to $a$ and $b^{\prime}$. Clearly no edge is seen in this third direction, the main feature again being a counterion absorption line. ${ }^{39}$

As final examples we show in Fig. 9 the $b^{\prime}$-axis edges in $(\mathrm{TMTSF})_{2} \mathrm{SbF}_{6}$ and (TMTSF) ${ }_{2} \mathrm{ClO}_{4}$. The $300-\mathrm{K} \quad b$-axis lattice constant in (TMTSF) ${ }_{2} \mathrm{SbF}_{6}$ is ${ }^{41} 7.728 \AA$, while in (TMTSF) ${ }_{2} \mathrm{ClO}_{4}$ it is ${ }^{42} 7.678 \AA$. The latter material has one of the most dense packings in the family, and it appears from Fig. 9 that this packing results in a significant blue shift of the transverse plasma edge, as might be expected.

For more quantitative estimates we have fitted the Drude model of Eq. (1) to the low-temperature edges of different materials. The sharp anion lines are modeled by 


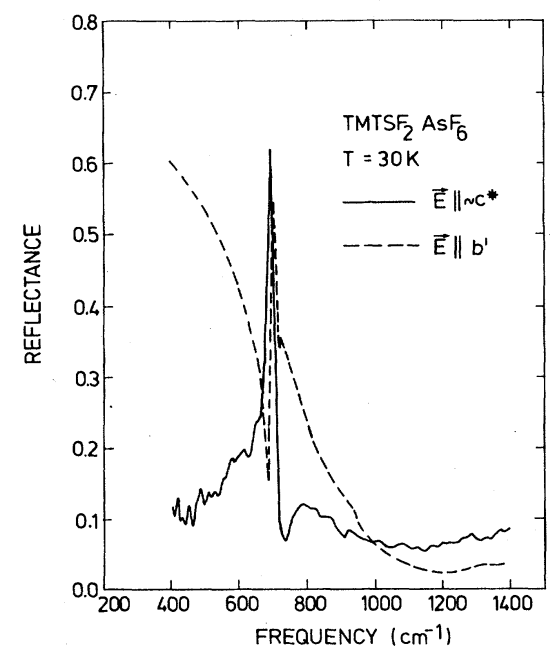

FIG. 8. Polarized reflectance for $\overrightarrow{\mathrm{E}} \| b^{\prime}$ and $\overrightarrow{\mathrm{E}} \sim \| c^{*}$ of $(\mathrm{TMTSF})_{2} \mathrm{AsF}_{6}$ at $T=30 \mathrm{~K}$. The frequency range is $400-1400$ $\mathrm{cm}^{-1}$.

including extra oscillators. We have assumed the background dielectric constant to be the same, $\epsilon_{\infty}=3.5$, in all cases, as estimated from the reflectance level in the (3000-4000)-cm $\mathrm{cm}^{-1}$ range. Results based on roomtemperature spectra show little change in oscillator strength, but are less accurate. In our earlier paper ${ }^{17}$ we used a simple effective mass model to estimate the transfer integral from the plasma frequencies. As pointed out by $\mathrm{Kwak},{ }^{43}$ the proper analysis also involves the chain axis transfer integral. For a simplified approach we consider a rectangular lattice with a tight-binding band

$$
\epsilon\left(k_{a}, k_{b}\right)=2 t_{a} \cos \left(k_{a} \frac{a}{2}\right)+2 t_{b} \cos \left(k_{b} b\right) .
$$

Assuming an open Fermi surface and $t_{b}<<t_{a}$ we obtain from Eq. (2)

$$
\omega_{p, b}^{2} \cong \frac{2 \sqrt{2}}{\pi} \frac{e^{2} b^{2}}{\epsilon_{0} \hbar^{2} V_{m}} \frac{t_{b}^{2}}{t_{a}}
$$

Thus apart from prefactors, the oscillator strength is suppressed by a factor of $t_{b} / t_{a}$. This is a result of the open Fermi surface, which produces many cancellations in the contributions to Eq. (2).

In Table II are given the resulting plasma frequencies and transfer integrals. Note that $t_{b}$ correlates well with the $b$-axis lattice constant. Note also that $t_{b} \simeq 0.1 t_{a}$ so that the assumption that $t_{b} \ll t_{a}$ may be questionable. Howev-

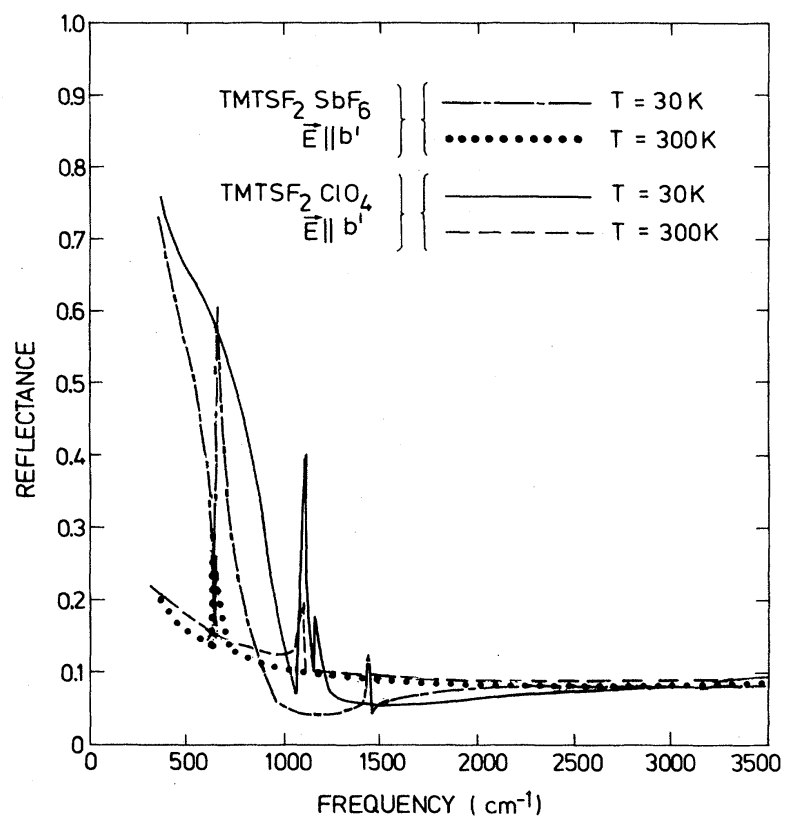

FIG. 9. Polarized reflectance for $\overrightarrow{\mathrm{E}} \| b^{\prime}$ of $(\mathrm{TMTSF})_{2} \mathrm{SbF}_{6}$ and (TMTSF) ${ }_{2} \mathrm{ClO}_{4}$ at $\mathrm{T}=30$ and $300 \mathrm{~K}$. The frequency range is $350-3500 \mathrm{~cm}^{-1}$.

er, the values given are in reasonable agreement with results from band-structure calculations reported by Grant. ${ }^{44}$ The implied band-structure anisotropy varies from 15 in (TMTSF) ${ }_{2} \mathrm{SbF}_{6}$ to 12 in (TMTSF) ${ }_{2} \mathrm{ClO}_{4}$. It should be noted that the transverse bandwidths are of order $800-1100 \mathrm{~K}$, so that the $b$-axis band curvature is significant at all temperatures of interest.

\section{Chain-axis intraband excitations}

Apart from slight shifts in the position of the plasma edge, the near-infrared chain-axis spectra of different materials look the same. As we shall see, this is not the case in the infrared region, where the effects of emv coupling and of metal-insulator transitions are evident.

The following materials will be discussed. (1) (TMTSF) ${ }_{2} \mathbf{P F}_{6}$ in its metallic phase, representing the highly conducting group of materials. (It was not possible to do measurements on this material below the metalinsulator transition at 12 K.) (2) (TMTTF) ${ }_{2} \mathrm{PF}_{6}$ as a typical example of a material with intermediate conductivity $\left[\sigma_{a}(300 \mathrm{~K})=20 \Omega^{-1} \mathrm{~cm}^{-1}\right]^{10}$ (3) $(\mathrm{TMTTF})_{2} \mathrm{Br}$ which is

TABLE II. Drude parameters, transfer integrals, and $b$-axis lattice constant in (TMTSF) ${ }_{2} X, \overrightarrow{\mathrm{E}}|| b^{\prime}$.

\begin{tabular}{cccccc}
\hline \hline $\boldsymbol{X}$ & $\epsilon_{c}$ & $\omega_{p}\left(\mathrm{~cm}^{-1}\right)$ & $\Gamma\left(\mathrm{cm}^{-1}\right)$ & $t_{b}(\mathrm{meV})$ & $b(\AA)$ \\
\hline $\mathrm{ClO}_{4}^{-}$ & 3.50 & 2020 & 250 & 24 & 7.678 (Ref. 42) \\
$\mathrm{SbF}_{6}{ }^{-}$ & & 1510 & 300 & 18 & 7.728 (Ref. 41) \\
$\mathrm{AsF}_{6}^{-}$ & & 1670 & 350 & 20 & 7.711 (Ref. 41) \\
$\mathrm{PF}_{6}^{-}$ & & 1830 & 500 & 22 & 7.711 (Ref. 13) \\
\hline \hline
\end{tabular}




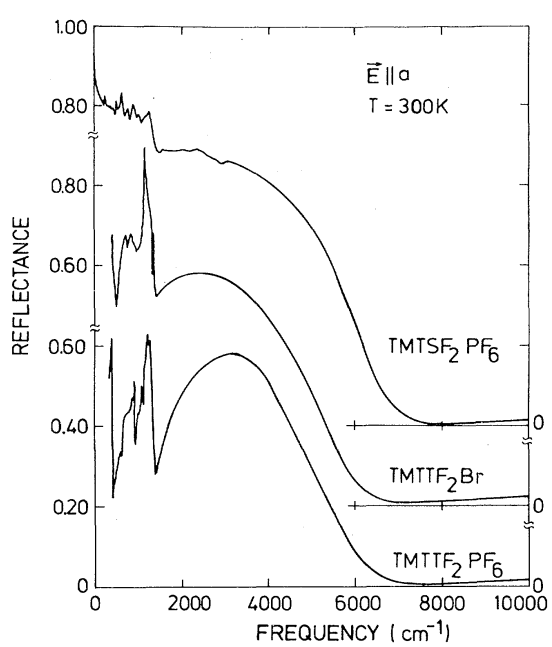

FIG. 10. Chain-axis reflectance of (TMTSF) ${ }_{2} \mathrm{PF}_{6}$, $(\mathrm{TMTTF})_{2} \mathrm{Br}$, and $(\mathrm{TMTTF})_{2} \mathrm{PF}_{6}$ at $T=300 \mathrm{~K}$. The frequency range is $10-10000 \mathrm{~cm}^{-1}$ for (TMTSF) ${ }_{2} \mathrm{PF}_{6}$, and $350-10000$ $\mathrm{cm}^{-1}$ for the (TMTTF) ${ }_{2} X$ salts.

the best metal in the (TMTTF $)_{2} X$ group $\left[\sigma_{a}(300 \mathrm{~K})=260\right.$ $\left.\Omega^{-1} \mathrm{~cm}^{-1}\right]{ }^{10}$ and which may be considered an intermediary between the two groups. Figure 10 shows the chainaxis polarized reflectance spectrum of these materials at $300 \mathrm{~K}$. The main differences clearly appear below 3000 $\mathrm{cm}^{-1}$, i.e., in the range of molecular vibrations. The material with lowest conductivity $\left[(\mathrm{TMTTF})_{2} \mathbf{P F}_{6}\right]$ has the strongest vibrational lines.

Figure 11 presents the frequency-dependent conductivi-

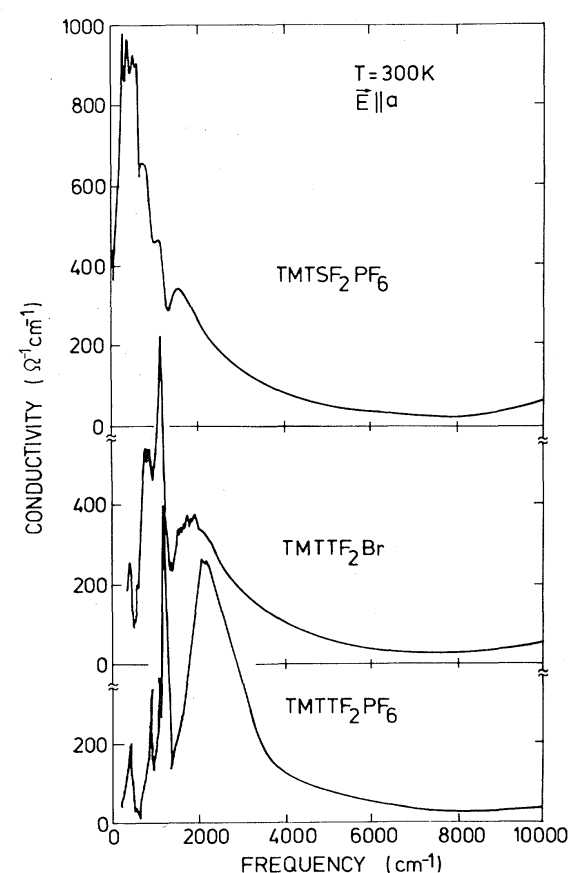

FIG. 11. Frequency-dependent chain-axis conductivity of $(\text { TMTSF })_{2} \mathrm{PF}_{6}$, (TMTTF $)_{2} \mathrm{Br}$, and (TMTTF $)_{2} \mathrm{PF}_{6}$ at $T=300 \mathrm{~K}$. The results are obtained by dispersion analysis of reflectance data. The frequency range is $10-10000 \mathrm{~cm}^{-1}$ for $(\mathrm{TMTSF})_{2} \mathrm{PF}_{6}$, and $350-10000 \mathrm{~cm}^{-1}$ for the (TMTTF) $)_{2} X$ salts. ties (determined by dispersion analysis ${ }^{34}$ ). The spectra can be viewed as broad peaks with fine structure of vibrational origin. These peaks are centered at 300, 1000, and $2200 \mathrm{~cm}^{-1}$ for $(\mathrm{TMTSF})_{2} \mathrm{PF}_{6}, \quad(\mathrm{TMTTF})_{2} \mathrm{Br}$, and (TMTTF) ${ }_{2} \mathrm{PF}_{6}$, respectively. Thus there seems to be a correlation between the position of the broad band and the metallic quality, for example, measured by the dc conductivity. For an ideal metal [described by the Drude model, Eq. (1)], the electronic band is centered at zero frequency. We note that for the materials in Fig. 11 the $\sigma(\omega)$ curves can easily be extrapolated to the proper dc values.

The physics behind the shift of oscillator strength to higher frequencies is not well understood. It appears that Coulomb correlations play a role, i.e., they give rise to a partial localization of the wave functions (as in a Mott insulator); consequently oscillator strength shifts away from zero frequency. These ideas have been discussed qualitatively by Hubbard. ${ }^{45}$ If, on the other hand, the electronic band is close to zero frequency, one may hope that models based on delocalized carriers [such as Eq. (2)] apply.

\section{ELECTRON-MOLECULAR-VIBRATION COUPLING EFFECTS}

We will now turn to a discussion of the vibrational features. It is of value to include data on (TMTSF) ${ }_{2} \mathrm{ReO}_{4}$, which has a sharp metal-insulator transition at $183 \mathrm{~K}$, related to anion ordering. ${ }^{9}$ The reflectance and conductivity of (TMTSF) ${ }_{2} \mathrm{ReO}_{4}$ are shown in Fig. 12 for temperatures above and below the metal-insulator transition. Note that the low-temperature spectrum is similar to that of $(\mathrm{TMTTF})_{2} \mathrm{PF}_{6}$ at $300 \mathrm{~K}$, even though $(\mathrm{TMTSF})_{2} \mathrm{ReO}_{4}$ at $40 \mathrm{~K}$ is believed to be an ordinary semiconductor, where the low conductivity is due to a gap at the Fermi level, caused by a $2 k_{F}$ potential. $^{9}$

Because most vibrational modes in the range of interest are polarized in the molecular plane, vibrational features polarized along the chain direction, i.e., almost perpendicular to this plane, are believed to be due to the Rice effect following from the emv coupling. ${ }^{46,47}$ This coupling is nonzero only for the totally symmetric $a_{g}$ modes and follows from the fact that the orbital energy levels in general will change when the size of the molecule is modulated.

\section{A. Vibrational mode assignments}

As basis for the discussion we list in Table III the calculated $a_{g}$ modes for TMTSF, as given by Bozio et al. ${ }^{21}$ We also include calculated coupling constants for the analogous modes in $\mathrm{TTF}^{+},{ }^{48}$ where appropriate.

In addition we show $\sigma(\omega)$ for (TMTSF) ${ }_{2} \mathrm{PF}_{6}$ and $(\mathrm{TMTTF})_{2} \mathbf{P F}_{6}$ (at $300 \mathrm{~K}$ ), and (TMTSF) ${ }_{2} \mathrm{ReO}_{4}$ (at $40 \mathrm{~K}$ ) on an expanded frequency scale in Fig. 13. (TMTTF) ${ }_{2} \mathrm{PF}_{6}$ and (TMTSF) ${ }_{2} \mathrm{ReO}_{4}$ both show a very strong resonance in the $1500-\mathrm{cm}^{-1}$ range. This band is believed to be due to the $\left(a_{g}, \omega_{4}\right)$ mode ${ }^{20,21}$ involving the central $\mathrm{C}=\mathrm{C}$ stretches. As seen in Table III calculations indicate a large coupling to the electrons for this mode. It also clearly has the effect of producing a broad minimum in $\sigma(\omega)$ for (TMTSF) ${ }_{2} \mathrm{PF}_{6}$. Other modes which are of unambiguous 

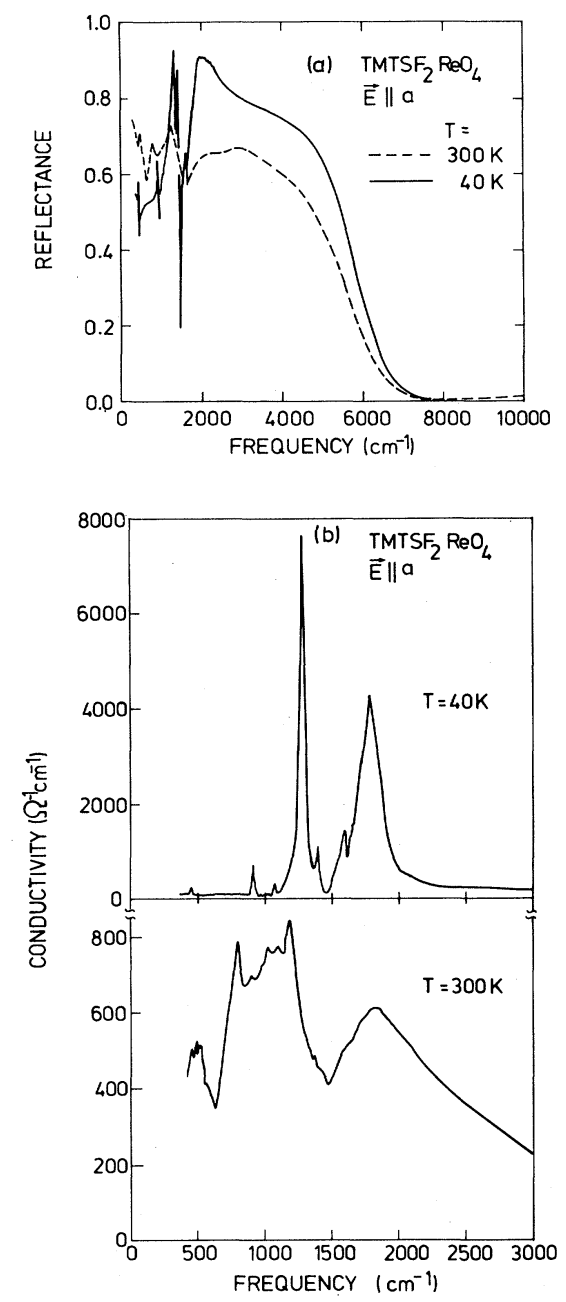

FIG. 12. Infrared properties of (TMTSF) ${ }_{2} \mathrm{ReO}_{4}$ above $(T=300 \mathrm{~K})$ and below $(T=40 \mathrm{~K})$ the metal-insulator transition at $T=183 \mathrm{~K}$. In (a) is shown the chain-axis reflectance in the range $400-10000 \mathrm{~cm}^{-1}$, and in (b) the resulting frequencydependent conductivities in the range $400-3000 \mathrm{~cm}^{-1}$. Notice the different conductivity scales at $T=300$ and $40 \mathrm{~K}$.

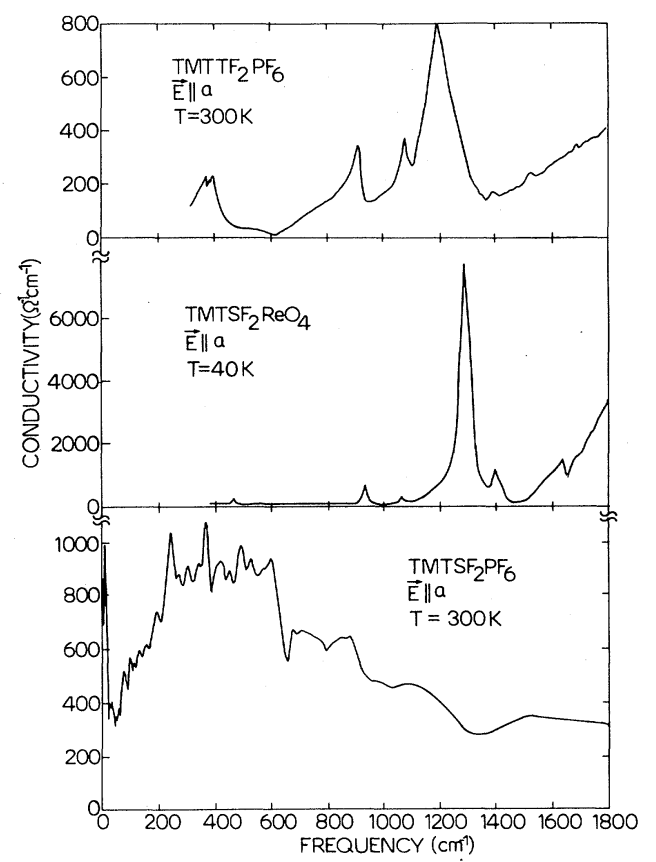

FIG. 13. Frequency-dependent chain-axis conductivity of (TMTTF $)_{2} \mathrm{PF}_{6}$ and $(\mathrm{TMTSF})_{2} \mathrm{PF}_{6}$ at $T=300 \mathrm{~K}$ and of (TMTSF) ${ }_{2} \mathrm{ReO}_{4}$ at $T=40 \mathrm{~K}$. The lower-frequency limit is 350 , 10 , and $400 \mathrm{~cm}^{-1}$ for the three materials, respectively, and the upper limit $1800 \mathrm{~cm}^{-1}$. Notice the different conductivity scales.

origin are the $\left(a_{g}, \omega_{3}\right)$ mode near $1600 \mathrm{~cm}^{-1}$, only resolved in (TMTSF) ${ }_{2} \mathrm{ReO}_{4}$, the $\left(a_{g}, \omega_{7}\right)$ mode near $1100 \mathrm{~cm}^{-1}$, and the $\left(a_{g}, \omega_{9}\right)$ mode at $430 \mathrm{~cm}^{-1}$ in (TMTSF) ${ }_{2} \mathrm{ReO}_{4}$. This latter mode involves the $\mathrm{C}-\mathrm{Se}$ bond and the corresponding vibration should be at a somewhat higher frequency ${ }^{20}$ in TMTTF $\left(552 \mathrm{~cm}^{-1}\right)$, where it does not seem to be resolved. Finally the strong mode at $390 \mathrm{~cm}^{-1}$ in (TMTTF $)_{2} \mathrm{PF}_{6}$ is the $\left(a_{g}, \omega_{10}\right)$ mode. $^{20}$

Another mode, seen at $910 \mathrm{~cm}^{-1}$ in both materials, should be the $\left(a_{g}, \omega_{8}\right)$ mode involving the methyl groups. This assignment may safely be made in the case of (TMTTF) ${ }_{2} \mathrm{PF}_{6}$, since the line is almost completely polar-

TABLE III. $A_{g}$ vibration modes for $\operatorname{TMTSF}^{0}($ Ref. 21$)$.

\begin{tabular}{rclc}
\hline \hline & $\begin{array}{c}\text { Calculated } \\
\text { frequency } \\
\left(\mathrm{cm}^{-1}\right)\end{array}$ & Dominating character & $\begin{array}{c}g_{\alpha} \text { for similar mode in } \\
\text { TTF }^{+}\left(\text {Ref. }^{4}\right) \\
\left(\mathrm{cm}^{-1}\right)\end{array}$ \\
\hline 1 & 2954 & C-H stretch & 90 \\
2 & 2862 & C-H stretch & 360 \\
3 & 1630 & C=C stetch, central plus ring & 940 \\
4 & 1548 & C=C stretch, central plus ring & \\
5 & 1440 & H-C-H bend & \\
6 & 1365 & C-C-H, H-C-H bend & \\
7 & 1087 & C-Se stretch, C-CH stretch, C-C-H bend & \\
8 & 929 & C-CH3 stretch, C-C-H bend & 630 \\
9 & 453 & C-Se stretch, C-CH - stretch & \\
10 & 299 & C-Se stretch, ring deformation & 40 \\
11 & 240 & Se-C-CH bend & 40 \\
12 & 146 & C-Se stretch, ring deformation & \\
\hline \hline
\end{tabular}


ized along the chain axis (see Fig. 7, where it is absent). For (TMTSF) ${ }_{2} \mathrm{ReO}_{4}$ an assignment is less unambiguous, because $\mathrm{ReO}_{4}{ }^{-}$is known to have an absorption band very close to this positon. ${ }^{21}$ However, because the $\mathrm{ReO}_{4}{ }^{-}$band is of comparable intensity to similar bands in $\mathrm{PF}_{6}{ }^{-}$at 835 $\mathrm{cm}^{-1}$, and because these $\mathrm{PF}_{6}{ }^{-}$bands are only weakly seen in the chain-axis polarization in (TMTTF $)_{2} \mathrm{PF}_{6}$, we conclude that most of the strength in the band is due to coupling with the $\left(a_{g}, \omega_{8}\right)$ mode. Furthermore, such a coupling may also account for the sudden drop in $\sigma(\omega)$ in (TMTSF) ${ }_{2} \mathrm{PF}_{6}$ at about the same frequency.

Similarly, the dip in the (TMTSF) ${ }_{2} \mathrm{ReO}_{4}$ spectrum at $1370 \mathrm{~cm}^{-1}$ may be due to coupling to the $\left(a_{g}, \omega_{6}\right)$ mode, which also involves the methyl groups. Bozio et al. ${ }^{21}$ argue that this coupling constant should be very small, because little electron density is expected near the methyl groups, and suggest that the dip could be caused by anharmonic interaction between the fully symmetric modes. In our opinion the spectra can be taken as evidence for considerable electronic coupling to the methyl group modes. It follows that there must be a considerable charge density near the groups, and this observation may provide a clue to understanding the large difference in transfer integrals between TTF (TSF) and TMTTF (TMTSF).

\section{B. Dimer model}

A proper theory for the emv coupling effects in these materials does not exist. However, it turns out that a model based on isolated dimers describes the results rather well. It should just be kept in mind that the resulting parameters may have to be reinterpreted at a later stage. In the dimer theory ${ }^{47}$ the dielectric function is described by

$$
\widetilde{\boldsymbol{\epsilon}}(\omega)=\epsilon_{\infty}+\frac{\omega_{p}^{2}}{\omega_{\mathrm{CT}}^{2}[1-D(\omega)]-\omega^{2}-i \omega \gamma_{e}} .
$$

Here $\omega_{p}^{2}$ is a measure of the strength in the charge-transfer band, $\omega_{\mathrm{CT}}$ determines its position in the absence of vibrational coupling, $\gamma_{e}$ is a relaxation rate, and $\epsilon_{\infty}$ is a background dielectric constant. The function $D(\omega)$ describes the modes and the coupling strengths:

$$
D(\omega)=\sum_{\alpha} \frac{\lambda_{\alpha} \omega_{\alpha}^{2}}{\omega_{\alpha}^{2}-\omega^{2}-i \omega \gamma_{\alpha}} .
$$

In Eq. (8), $\omega_{\alpha}$ and $\gamma_{\alpha}$, respectively, are the bare frequency and inverse lifetime of mode $\alpha$, and $\lambda_{\alpha}$ is a dimensionless coupling constant. This quantity is related to the coupling constant $g_{\alpha}$ by

$$
\lambda_{\alpha}=\frac{4 \epsilon_{0}}{n e^{2} a^{2}} \frac{\omega_{p}^{2}}{\omega_{\mathrm{CT}}^{2}} \frac{g_{\alpha}^{2}}{\omega_{\alpha}},
$$

where $n$ is the dimer density, and $a$ is the spacing between molecules.

We have fitted this model to the reflectance of $(\mathrm{TMTTF})_{2} \mathrm{PF}_{6}$ at $300 \mathrm{~K}$ and $(\mathrm{TMTSF})_{2} \mathrm{ReO}_{4}$ at $40 \mathrm{~K}$. The quality of the fits may be seen in Figs. 14 and 15 . The parameters found are listed in Table IV with the assignments described above.

Although the results look fairly reasonable, it seems

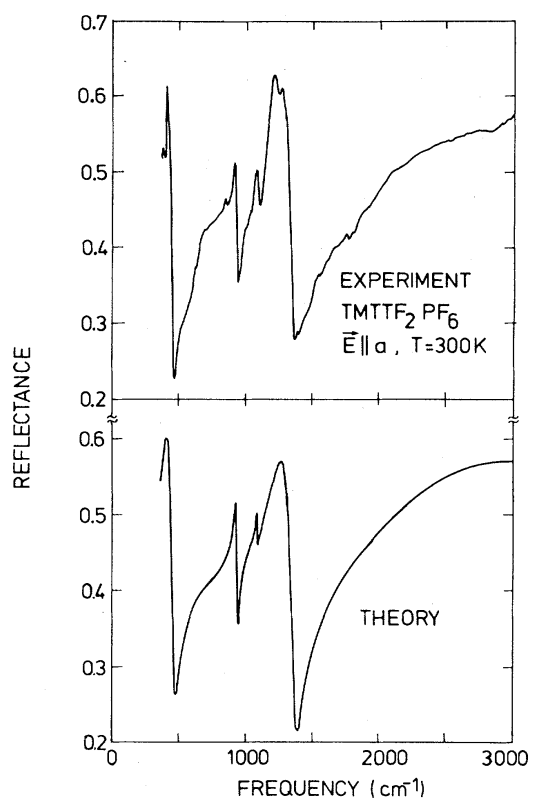

FIG. 14. Chain-axis reflectance of (TMTTF) ${ }_{2} \mathbf{P F}_{6}$ at $T=300$ $\mathrm{K}$ as measured and as calculated by fitting the dimer model to the data. The frequency range is $350-3000 \mathrm{~cm}^{-1}$.

that both the predicted coupling constants and the frequency shifts are too small. For example, in (TMTTF) $)_{2} \mathrm{PF}_{6}$ the $\left(a_{g}, \omega_{4}\right)$ mode has $g_{4}=300 \mathrm{~cm}^{-1}$ as compared with the calculated value of $940 \mathrm{~cm}^{-1}$ for TTF $^{+}$(Table III). The mode is located at $1185 \mathrm{~cm}^{-1}$ and the bare frequency should be $1378 \mathrm{~cm}^{-1}$ according to Table IV. Torrance et al. ${ }^{49}$ found by Raman spectroscopy

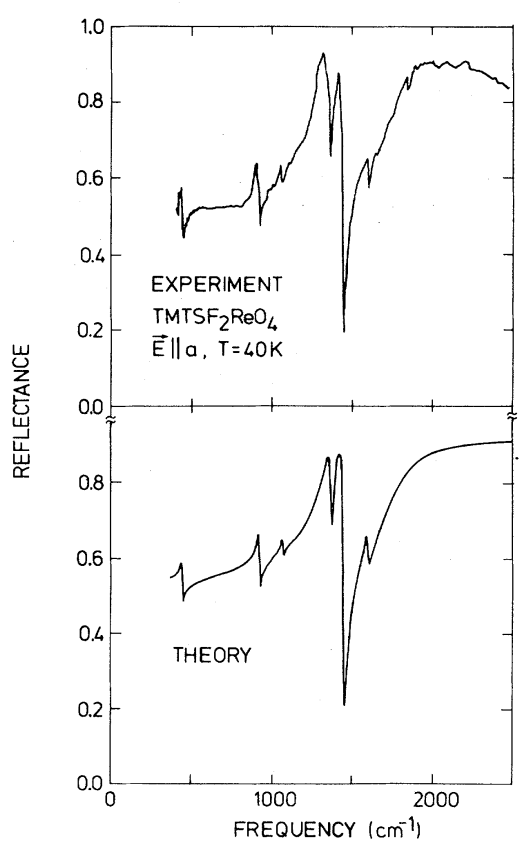

FIG. 15. Chain-axis reflectance of (TMTSF) ${ }_{2} \mathrm{ReO}_{4}$ at $T=40$ $\mathrm{K}$ as measured and as calculated by fitting the dimer model to the data. The frequency range is $400-2500 \mathrm{~cm}^{-1}$. 
TABLE IV. Dimer charge oscillator parameters for (TMTTF) $)_{2} \mathbf{P F}_{6}(300 \mathrm{~K})$ and (TMTSF) ${ }_{2} \operatorname{ReO}_{4}(40 \mathrm{~K})$.

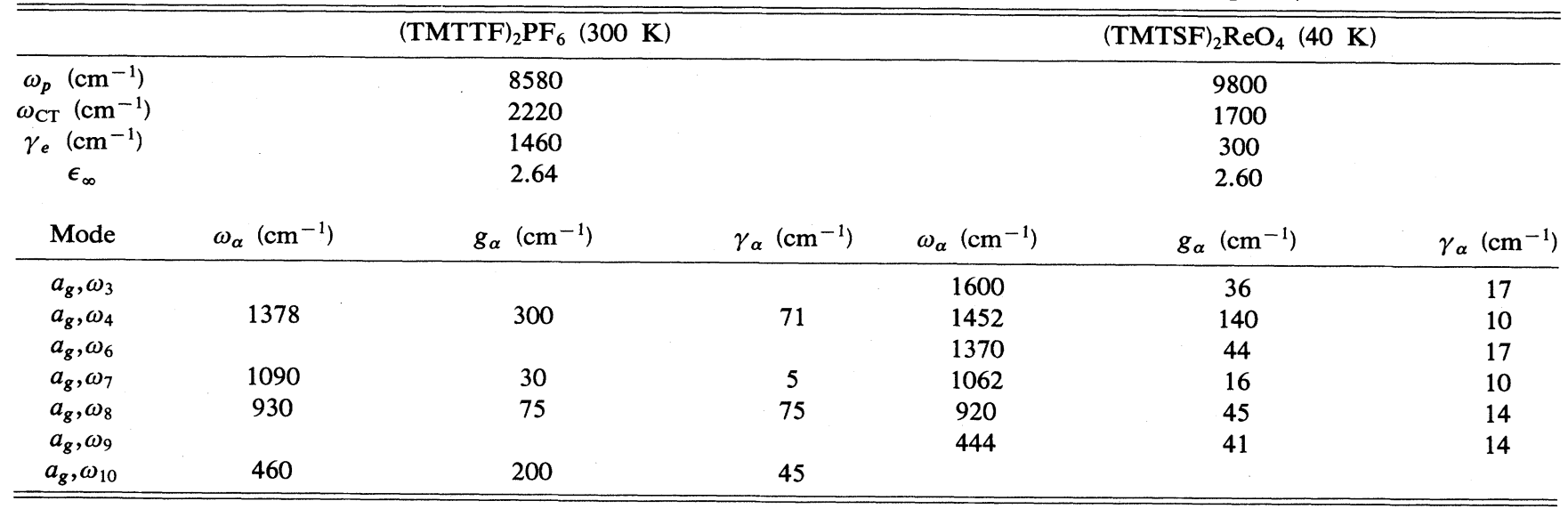

that $\omega_{4}=1476 \mathrm{~cm}^{-1}$ in another TMTTF compound with the same molecular charge. Bozio et al. ${ }^{20}$ have also determined coupling constants from the dimer model. They base their calculation on the frequency shifts predicted by the dimer model, and therefore obtain somewhat different values: For (TMTTF) ${ }_{2} \mathrm{BF}_{4}$ they report $g_{4}=670 \mathrm{~cm}^{-1}$ and $g_{10}=480 \mathrm{~cm}^{-1}$.

In conclusion we state that although the interpretation of the model described by Eq. (7) may be questioned, it seems clear that it describes the real spectra quite well. It is actually also possible by adjusting $\omega_{\mathrm{CT}}$ to lower values to reproduce the qualitative features of, for example, the $(\mathrm{TMTTF})_{2} \mathrm{Br}$ and $(\mathrm{TMTSF})_{2} \mathrm{PF}_{6}$ spectra.

\section{CHAIN-AXIS CONDUCTIVITY OF (TMTSF) ${ }_{2} \mathrm{PF}_{6}$}

\section{A. Results}

We now turn to the $a$-axis properties of (TMTSF) ${ }_{2} \mathrm{PF}_{6}$ at three temperatures: 300,100 , and $25 \mathrm{~K}$ and concentrate on the range $0-1200 \mathrm{~cm}^{-1}$. Figure 16 shows the re-

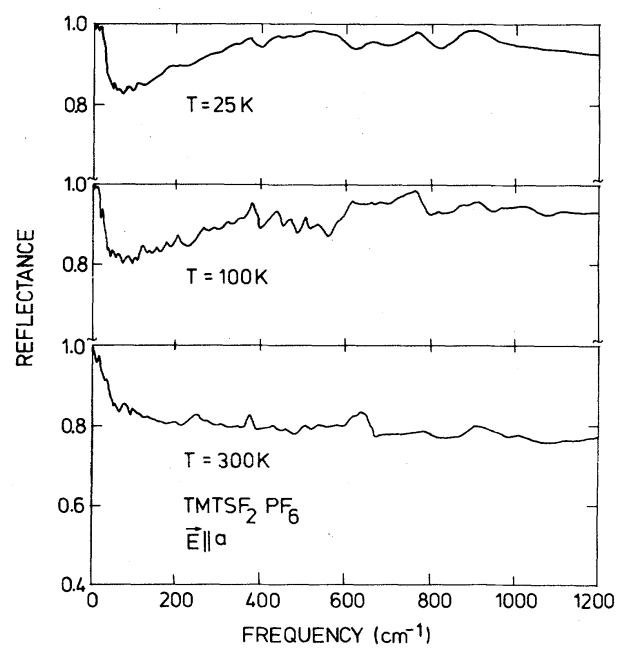

FIG. 16. Chain-axis reflectance of (TMTSF) ${ }_{2} \mathrm{PF}_{6}$ for $T=25$, 100 , and $300 \mathrm{~K}$. The frequency range is $10-1200 \mathrm{~cm}^{-1}$. flectance starting at $10 \mathrm{~cm}^{-1}$. Apart from the fine structure, most of which is unidentified, two features are remarkable. (1) $R$ goes to unity at low frequencies in a non-Drude manner. The Drude model predicts $1-R \sim \sqrt{\omega}$ for $\omega \ll \Gamma$, while we find in the lowtemperature data a reflectance near unity extending to 20 $\mathrm{cm}^{-1}$ and then a sudden drop to $80-82 \%$. (2) The 25and $100-\mathrm{K}$ reflectance rises again at higher frequencies. At $25 \mathrm{~K}$ there is a range from 400 to $900 \mathrm{~cm}^{-1}$ where $R$ is 95-99\%.

These features have important consequences for the frequency-dependent conductivity and dielectric function, which we have calculated by dispersion analysis, and present in Figs. 17 and 18. The basic features are clear:

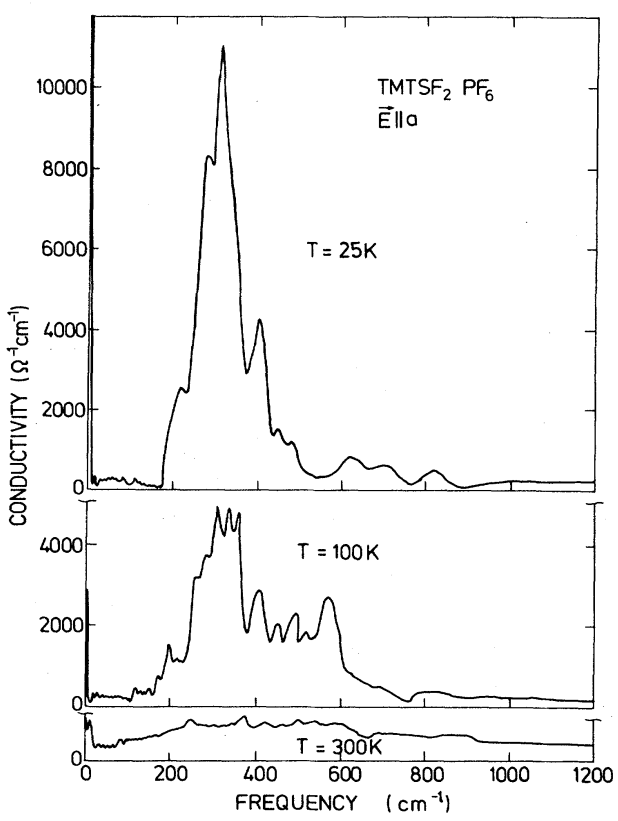

FIG. 17. Frequency-dependent chain-axis conductivity of (TMTSF) ${ }_{2} \mathbf{P F}_{6}$ for $T=25,100$, and $300 \mathrm{~K}$. The results are obtained by dispersion analysis of reflectance data. The valid frequency range is $10-1200 \mathrm{~cm}^{-1}$. However, the curves are extrapolated towards the known dc values below $10 \mathrm{~cm}^{-1}$. 


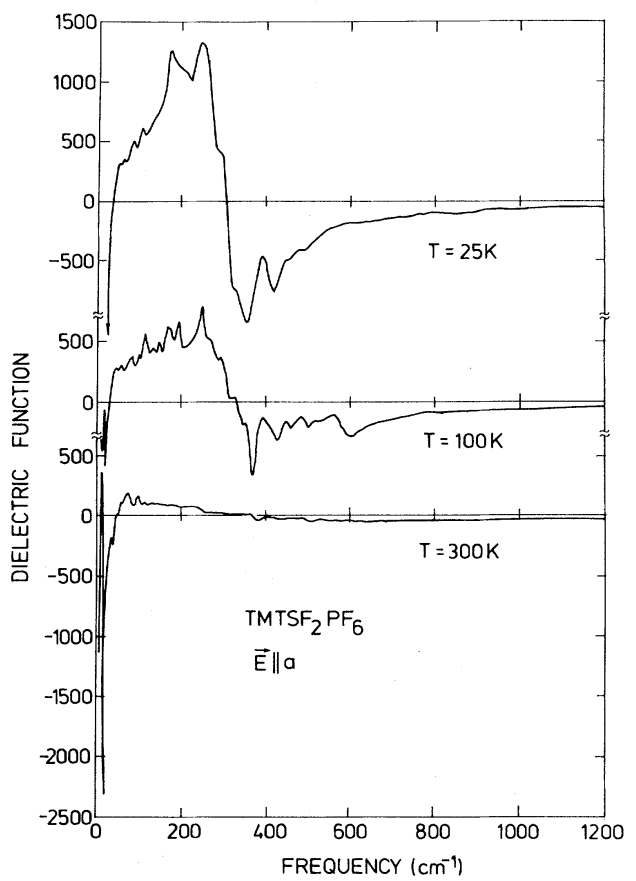

FIG. 18. Real part of the chain-axis dielectric function of (TMTSF) ${ }_{2} \mathbf{P F}_{6}$ for $T=25,100$, and $300 \mathrm{~K}$. The results are obtained by dispersion analysis of reflectance data. The frequency range is $10-1200 \mathrm{~cm}^{-1}$.

A dominant peak in $\sigma_{a}(\omega)$ around $300 \mathrm{~cm}^{-1}$ grows up with decreasing temperature. This growth is accompanied by a suppression of the far-infrared conductivity. The upturn towards the de values indicated in Fig. 17 is implied by, but not contained in the data. At $25 \mathrm{~K}$ there is virtually an excitation gap up to $180 \mathrm{~cm}^{-1}$. The distribution of oscillator strength [i.e., the area under the $\sigma_{a}(\omega)$ curves] can be estimated from the data. At $25 \mathrm{~K}$ about $70 \%$ of the total strength (as given by $\omega_{p}$ ) is contained in the 300 $\mathrm{cm}^{-1}$ peak. Furthermore, it is possible to make a reasonable estimate of the strength in the narrow mode near $\mathrm{dc}$, if $\widetilde{\boldsymbol{\epsilon}}(\omega)$ is modeled as a sum of three terms:

$$
\widetilde{\boldsymbol{\epsilon}}(\omega)=\epsilon_{\infty}+\frac{\beta \omega_{p}^{2}}{\omega_{0}^{2}-\omega^{2}-i \omega \Gamma}-\frac{\Omega_{p}^{2}}{\omega(\omega+i \gamma)},
$$

where the second and the third terms represent the dominant peak and the narrow mode, respectively.

At $25 \mathrm{~K}, \beta=0.70$ (see above) and $\omega_{o} \cong 300 \mathrm{~cm}^{-1}$. Thus at $31 \mathrm{~cm}^{-1}$, where $\epsilon_{1}(\omega)$ crosses zero (see Fig. 18), the first two terms represent an approximately frequencyindependent background dielectric constant of about 800 . The last term will cancel this contribution at $31 \mathrm{~cm}^{-1}$ for $\Omega_{p} \cong 900 \mathrm{~cm}^{-1}$. Finally with $^{3} \quad \sigma_{\mathrm{dc}}(25 \quad \mathrm{~K})=5 \times 10^{4}$ $\Omega^{-1} \mathrm{~cm}^{-1}$, the halfwidth $\gamma$ may be estimated to $0.3 \mathrm{~cm}^{-1}$, which corresponds roughly to $10 \mathrm{GHz}$. Thus the results indicate that $\sigma(\omega)$ should fall rather rapidly through the microwave range. $35-\mathrm{GHz}$ data ${ }^{3}$ do in fact yield a somewhat higher resistivity than dc data in this temperature range, and perhaps more significantly, $\sigma_{35 \mathrm{GHz}}$ increases more slowly with decreasing temperature below $50 \mathrm{~K}$ than does $\sigma_{\mathrm{dc}}$.

\section{B. Background}

In order to interpret the data, it is useful to describe shortly the far-infrared properties of other highly conducting organics. The far-infrared properties of (TMTSF) ${ }_{2} \mathrm{ClO}_{4}$ have recently been measured by $\mathrm{Ng}$ et $a .^{23}$ and by Challener et al. ${ }^{24}$ (TMTSF) ${ }_{2} \mathrm{ClO}_{4}$ has no metal-insulator transition, but rather a superconducting transition at $1.3 \mathrm{~K}^{5}$ The reflectance at helium temperatures is high $(96-99 \%)$ up to $30 \mathrm{~cm}^{-1}$ and then falls abruptly to $90 \%$. Although this behavior is somewhat similar to (TMTSF) ${ }_{2} \mathrm{PF}_{6}$, there is considerable structure below $30 \mathrm{~cm}^{-1}$ : $\sigma_{a}(\omega)$ displays a double-peak structure which, however, rapidly disappears with increasing temperature. With the applied extrapolations $\sigma_{a}(\omega)$ rises gradually from a minimum at $30 \mathrm{~cm}^{-1}$ on going to higher frequencies. Thus the sudden increase in (TMTSF) ${ }_{2} \mathrm{PF}_{6}$ at $180 \mathrm{~cm}^{-1}$ is absent.

The far-infrared conductivity of TTF-TCNQ (Ref. 27) at the conductivity maximum $(T=60 \mathrm{~K})$ also shows some similarities with that of (TMTSF) ${ }_{2} \mathrm{PF}_{6}$. There is a peak in $\sigma(\omega)$ at $300 \mathrm{~cm}^{-1}$, usually attributed to a fluctuating Peierls gap, ${ }^{27}$ a rather low conductivity in the (20-200)$\mathrm{cm}^{-1}$ range, and then a rise towards the dc value. The low-frequency rise is thought to be due to charge-densitywave contributions to the conductivity.

\section{Spin-density-wave model}

Returning to the (TMTSF) ${ }_{2} \mathrm{PF}_{6}$ data, a comparison with (TMTSF) ${ }_{2} \mathrm{ClO}_{4}$ hints that the difference in the farinfrared properties is related to the known differences in physical properties: (TMTSF) ${ }_{2} \mathrm{ClO}_{4}$ is known to have less tendency towards a spin-density-wave (SDW) instability than (TMTSF) ${ }_{2} \mathrm{PF}_{6}$, where a SDW ordered state is found below $12 \mathrm{~K}^{6}$ Thus we may assume that the observed gap is a threshold for SDW excitations. These should have a sharp gap $^{50}$ as observed. Under this assumption, the behavior is analogous to that of TTF-TCNQ: The narrow contribution to $\sigma(\omega)$ at dc is due to current carrying, sliding SDW's (Ref. 51) and the single-particle contributions are suppressed by a pseudogap, originating from the spindensity waves. These SDW's have a wave vector which just spans the Fermi surface. This interpretation assumes that there are important SDW fluctuations above the actual transition temperature. In spite of the rather large $b$ axis transfer integral, the fluctuations appear to be rather one dimensional: The absence of a pseudogap along $b^{\prime}$ (cf. Fig. 6) suggests that the transverse correlation length is limited to a few lattice constants.

That magnetic order may exist in the conducting state in these materials is independently confirmed by the observation of antiferromagnetic resonance in (TMTSF) ${ }_{2} \mathrm{ClO}_{4}{ }^{52}$ This shows that a SDW structure may be present without suppressing the dc conductivity. In a sense we are speaking about an itinerant antiferromagnet. But the real antiferromagnetism as detected in the macroscopic susceptibility ${ }^{6}$ does not occur until full threedimensional order is established.

Pursuing the idea of important SDW fluctuations we may estimate the amplitude of the SDW's from the gap 
size, using the simplest possible mean-field theory, which predicts $^{53}$

$$
E_{g}=2 \epsilon_{F} \exp \left[-1 / \rho_{\uparrow}\left(\epsilon_{F}\right) \widetilde{U}\right]=2 \frac{\mu}{\mu_{B}} \widetilde{U}
$$

Here $\mu / \mu_{B}$ is the amplitude (as fraction of the Bohr magneton), $\rho_{\uparrow}\left(\epsilon_{F}\right)$ is the single-spin density of states at the Fermi level $\epsilon_{F}$, and $\widetilde{U}$ is an effective Coulomb repulsion. With $t_{a}=0.28 \mathrm{eV}$ we have $\epsilon_{F}=0.16 \mathrm{eV}$ and $\rho_{\uparrow}\left(\epsilon_{F}\right)=0.80$ $\mathrm{eV}^{-1}$. The observed gap at $180 \mathrm{~cm}^{-1}$ corresponds to $E_{g}=22 \mathrm{meV}$. Then Eq. (8) gives $\widetilde{U}=0.47 \mathrm{eV}$ and $\mu / \mu_{B}=2.3 \%$. Other estimates of $\mu / \mu_{B}$ span this value, ranging from ${ }^{7} 0.6 \%$ to ${ }^{8} 10-20 \%$.

Finally we note that $E_{g}=22 \mathrm{meV}$ is the direct, optical gap. The observed thermodynamic gap of $4 \mathrm{meV}$ (Ref. 54) in the semiconducting state indicates that below $12 \mathrm{~K}$, the SDW wave vector does not nest the full Fermi surface. Incomplete nesting has been discussed in detail by $\mathrm{Kwak}^{55}$ in connection with the occurence of quantum oscillations. In view of the large transverse coupling, it will lead to a considerable reduction of the thermodynamic gap, even if the direct gap is unchanged.

\section{CONCLUSIONS}

In summary, we have made extensive measurements of the optical properties of (TMTSF) ${ }_{2} X$ and (TMTTF) ${ }_{2} X$ compounds. The data have been analyzed to obtain information about the electronic properties of these materials.

We have obtained three independent indications that the highest occupied molecular orbital on $(\text { TMTSF) })^{0}$ or (TMTSF) $^{+}$differs qualitatively from the corresponding TTF and TSF orbital: (1) The visible spectrum shows new structure, (2) the chain-axis transfer integral is unusually large $\left(t_{a} \cong 0.25 \mathrm{eV}\right.$ at $\left.300 \mathrm{~K}\right)$ for a slipped overlap, and (3) the anomalous emV spectra indicate considerable charge density near the methyl groups. This latter conclusion also holds for TMTTF.

The chain-axis infrared spectra for a series of compounds in the (TMTSF) ${ }_{2} X$ and (TMTTF) ${ }_{2} X$ family show a charge-transfer band. The position of this band correlates with the metallic character: a low-peak position corresponds to a high-dc conductivity. The vibronic features can be modeled very well using a simple dimer charge oscillator model.

The conducting (TMTSF) $)_{2} X$ compounds display the unique feature of a $b$-axis plasma edge near $1000 \mathrm{~cm}^{-1}$. Bandwidth anisotropies are found to be in the range 12-15 for different materials, and indicate open Fermi surfaces with significant two-dimensional character.

Finally, in (TMTSF) ${ }_{2} \mathbf{P F}_{6}$ the charge-transfer band is centered at $300 \mathrm{~cm}^{-1}$ and sharpens up with decreasing temperature. At $25 \mathrm{~K}$ a virtual gap appears at $180 \mathrm{~cm}^{-1}$. This gap is interpreted as an onset of excitations in a spin-density-wave structure, and implies that the dc conductivity is dominated by spin-density-wave transport. Analysis suggests a small-amplitude spin-density wave of $0.02 \mu_{B}$ at $25 \mathrm{~K}$.

\section{ACKNOWLEDGMENTS}

We acknowledge informative discussions with J. B. Torrance, T. Timusk, R. Bozio, and P. M. Grant. We thank I. Johannsen for supplying the (TMTTF) ${ }_{2} \mathrm{Br}$ crystals. The collaborative aspect of the work has been supported by the NATO Research Grants Programme. One of us (C.S.J.) acknowledges support from the Danish Natural Science Research Council and from the Royal Danish Academy of Sciences and Letters through a Niels Bohr Scholarship.
${ }^{1}$ Proceedings of the International Conference on Low Dimensional Conductors, Boulder, Colorado, 1981 [Mol. Cryst. Liq. Cryst. $\underline{77}$ (1981); $\underline{79}, \underline{81}, \underline{83}, \underline{85}, \underline{86}$ (1982)].

${ }^{2}$ Proceedings of the International Conference on Low Dimensional Conductors and Superconductors, Les Arcs, 1982 [J. Phys. (Paris) Colloq. $\underline{3}$ (1983)].

${ }^{3}$ K. Bechgaard, C. S. Jacobsen, K. Mortensen, H. J. Pedersen, and N. Thorup, Solid State Commun. 33, 1119 (1980).

${ }^{4}$ D. Jerome, A. Mazaud, M. Ribault, and K. Bechgaard, J. Phys. (Paris) Lett. 41, L95 (1980).

${ }^{5}$ K. Bechgaard, K. Carneiro, M. Olsen, F. B. Rasmussen, and C. S. Jacobsen, Phys. Rev. Lett. 46, 852 (1981).

${ }^{6} \mathrm{~K}$. Mortensen, Y. Tomkiewicz, T. D. Schultz, and E. M. Engler, Phys. Rev. Lett. 46, 1234 (1981).

${ }^{7}$ A. Andrieux, D. Jerome, and K. Bechgaard, J. Phys. (Paris) Lett. 42, L87 (1981).

${ }^{8}$ J. B. Torrance, H. J. Pedersen, and K. Bechgaard, Phys. Rev. Lett. 49 , 881 (1982).

${ }^{9}$ C. S. Jacobsen, H. J. Pedersen, K. Mortensen, G. Rindorf, N. Thorup, J. B. Torrance, and K. Bechgaard, J. Phys. C $\underline{15}$, 2651 (1982).

10P. Delhaes, C. Coulon, J. Amiell, S. Flandrois, E. Toreilles, J. M. Fabre, and L. Giral, Mol. Cryst. Liq. Cryst. 50, 43 (1979).
${ }^{11}$ S. S. P. Parkin, F. Creuzet, M. Ribault, D. Jerome, K. Bechgaard, and J. M. Fabre, Mol. Cryst. Liq. Cryst. 79, 249 (1982).

12J. P. Pouget, R. Moret, R. Comes, and K. Bechgaard, J. Phys. (Paris) Lett. 42, L543 (1981).

${ }^{13}$ N. Thorup, G. Rindorf, H. Soling, and K. Bechgaard, Acta Crystallogr. Sect. B 37, 1236 (1981).

${ }^{14}$ B. Liautard, S. Peytavin, G. Brun, and M. Maurin, J. Phys. (Paris) 43, 1454 (1982).

${ }^{15}$ T. J. Kistenmacher, T. E. Phillips, and D. O. Cowan, Acta Crystallogr. Sect. B $\underline{30}, 763$ (1974).

16V. J. Emergy, R. Bruinsma, and S. Barisic, Phys. Rev. Lett. 48, 1039 (1982).

${ }^{17}$ C. S. Jacobsen, D. B. Tanner, and K. Bechgaard, Phys. Rev. Lett. $\underline{46}, 1142$ (1981).

${ }^{18}$ C. S. Jacobsen, D. B. Tanner, and K. Bechgaard, Mol. Cryst. Liq. Cryst. 79, 25 (1982); J. Phys. (Paris) Colloq. 조 C-859 (1983).

${ }^{19}$ K. Kikuchi, Y. Ikemoto, K. Yakushi, H. Kuroda, and K. Kobayashi, Solid State Commun. 42, 433 (1982).

${ }^{20}$ R. Bozio, M. Meneghetti, and C. Pecile, J. Chem. Phys. 76, 5785 (1982).

${ }^{21}$ R. Bozio, C. Pecile, K. Bechgaard, F. Wudl, and D. Nalewa- 
jek, Solid State Commun. 41, 905 (1982).

${ }^{22}$ H. K. Ng, T. Timusk, J. M. Delrieu, D. Jerome, K. Bechgaard, and J. M. Fabre, J. Phys. (Paris) Lett. 433, L513 (1982).

${ }^{23}$ H. K. Ng, T. Timusk, and K. Bechgaard, J. Phys. Paris Colloq. $\underline{3}, \mathrm{C}-867$ (1983).

${ }^{24}$ W. A. Challener, P. L. Richards, and R. L. Greene, J. Phys. Paris Colloq. $\underline{3}$, C-873 (1983).

${ }^{25}$ H. W. Helberg, J. Phys. Paris Colloq. 3 , C-1021 (1983).

${ }^{26}$ L. B. Coleman, Ph.D. thesis, University of Pennsylvania,1975 (unpublished).

${ }^{27}$ D. B. Tanner and C. S. Jacobsen, Mol. Cryst. Liq. Cryst. $\underline{85}$, 137 (1982).

${ }^{28}$ P. M. Grant, R. L. Greene, G. C. Wrighton, and G. Castro, Phys. Rev. Lett. 1ㅣ, 1311 (1973).

${ }^{29}$ R. Zahradnik, P. Carsby, S. Hünig, G. Kiesslich, and D. Scheutzow, Int. J. Sulfur Chem. C $\underline{6}, 109$ (1971).

${ }^{30}$ R. Gleiter, M. Kobayashi, J. Spanget-Larsen, J. P. Ferraris, A. N. Bloch, K. Bechgaard, and D. O. Cowan, Ber. Bunsenges. Phys. Chem. 79, 1218 (1975).

${ }^{31}$ J. B. Torrance, B. A. Scott, B. Welber, F. B. Kaufman, and P. E. Seiden, Phys. Rev. B 19, 730 (1979).

${ }^{32}$ T. Sugano, K. Yakushi, and H. Kuroda, Bull. Chem. Soc. Jpn. 51, 1041 (1978).

${ }^{33}$ Y. Cao, K. Yakushi, and H. Kuroda, Solid State Commun. 35, 739 (1980).

${ }^{34}$ F. Wooten, Optical Properties of Solids (Academic, New York, 1972).

${ }^{35} \mathrm{~K}$. Mortensen, Solid State Commun. 44, 643 (1982).

${ }^{36}$ C. S. Jacobsen (unpublished).

${ }^{37}$ S. Etemad, T. Penney, E. M. Engler, B. A. Scott, and P. E. Seiden, Phys. Rev. Lett. 34, 741 (1975).

${ }^{38}$ B. Welber, P. E. Seiden, and P. M. Grant, Phys. Rev. B 18 ,
2692 (1978).

${ }^{39}$ G. M. Begun and A. C. Rutenberg, Inorg. Chem. 6, 2212 (1967).

${ }^{40}$ C. S. Jacobsen, K. Mortensen, M. Weger, and K. Bechgaard, Solid State Commun. 38,423 (1981).

${ }^{41} \mathrm{~N}$. Thorup (unpublished).

${ }^{42}$ K. Bechgaard, K. Carneiro, F. B. Rasmussen, M. Olsen, G. Rindorf, C. S. Jacobsen, H. J. Pedersen, and J. C. Scott, J. Am. Chem. Soc. 103, 2440 (1981).

43J. F. Kwak, Phys. Rev. B $\underline{26}, 4789$ (1982).

${ }^{44}$ P. M. Grant, Phys. Rev. B 266, 6888 (1982).

45 J. Hubbard, Phys. Rev. B 17, 494 (1978).

46M. J. Rice, Phys. Rev. Lett. 그, 36 (1976); Solid State Commun. 31, 93 (1979).

${ }^{47}$ M. J. Rice, V. M. Yartsev, and C. S. Jacobsen, Phys. Rev. B 21, 3437 (1980).

${ }^{48}$ N. O. Lipari, M. J. Rice, C. B. Duke, R. Bozio, A. Girlando, and C. Pecile, Int. J. Quantum Chem. Symp. 11, 583 (1977).

${ }^{49}$ J. B. Torrance, J. J. Mayerle, V. Y. Lee, R. Bozio, and C. Pecile, Solid State Commun. $\underline{38}, 1165$ (1981).

${ }^{50}$ E. W. Fenton and G. C. Psaltakis, Solid State Commun. 47, 767 (1983).

${ }^{51}$ P. A. Lee, T. M. Rice, and P. W. Anderson, Solid State Commun. 14, 703 (1974).

${ }^{52}$ W. M. Walsh, Jr., F. Wudl, E. Aharon-Shalom, L. W. Rupp. Jr., J. M. Vandenburg, K. Andres, and J. B. Torrance, Phys. Rev. Lett. 49, 885 (1982).

53J. C. Scott, Mol. Cryst. Liq. Cryst. 79, 49 (1982).

${ }^{54}$ P. M. Chaikin, P. Haen, E. M. Engler, and R. L. Greene, Phys. Rev. B 24, 7155 (1981).

55J. F. Kwak, Phys. Rev. B $2 \underline{8}, 3277$ (1983). 